\title{
Inhibition of Caspase-Mediated Apoptosis by Peroxynitrite in Traumatic Brain Injury
}

\author{
Anthony Lau, ${ }^{1,2}$ Mark Arundine, ${ }^{1,2}$ Hong-Shuo Sun, ${ }^{1,2}$ Michael Jones, ${ }^{1,2}$ and Michael Tymianski1 ${ }^{1,2}$ \\ ${ }^{1}$ Division of Applied and Interventional Research, Toronto Western Research Institute, Toronto, Ontario, Canada M5T 2S8, and Departments of \\ ${ }^{2}$ Physiology and ${ }^{3}$ Neurosurgery, University of Toronto, Toronto, Ontario, Canada M5S 1A8
}

In traumatic brain injury (TBI), neurons surviving the primary insult may succumb through poorly understood secondary mechanisms. In vitro, cortical neurons exposed to stretch injury exhibited enhanced vulnerability to NMDA, apoptotic-like DNA fragmentation, peroxynitrite (PN) formation, and cytoplasmic cytochrome $c$ accumulation. Surprisingly, caspase-3 activity was undetectable by both immunoblotting and fluorogenic activity assays. Therefore, we hypothesized that PN directly inhibits caspases in these neurons. Consistent with this, stretch injury in cultured neurons elicited tyrosine nitration of procaspase-3, but not caspase- 9 or Apaf-1, suggesting a direct interaction of $\mathrm{PN}$ with caspase-3. In an ex vivo system, $\mathrm{PN}$ inhibited the activity of caspase-3, and this inhibition was reversible with the addition of the sulfhydryl reducing agent dithiothreitol, indicating that PN inhibits caspases by cysteinyl oxidation. Moreover, in cultures, the PN donor 3-morpholinosydnonimine (SIN-1) blocked staurosporine-induced caspase-3 activation and its downstream effects including PARP-1 [poly-(ADP-ribose) polymerase-1] cleavage and phosphotidylserine inversion, suggesting that peroxynitrite can inhibit caspase-3-mediated apoptosis. To examine these mechanisms in vivo, rats were exposed to a lateral fluid percussion injury (FPI). FPI caused increased neuronal protein nitration that colocalized with TUNEL staining, indicating that PN was associated with neurodegeneration. Caspase-3 activity was inhibited in brain lysates harvested after FPI and was restored by adding dithiothreitol. Our data show that caspase-mediated apoptosis is inhibited in neurons subjected to stretch in vitro and to TBI in vivo, mostly because of cysteinyl oxidation of caspase- 3 by PN. However, this is insufficient to prevent cell death, indicating that the TBI therapy may, at a minimum, require a combination of both anti-apoptotic and anti-oxidant strategies.

Key words: peroxynitrite; traumatic brain injury; caspase-3; apoptosis; nitric oxide; reactive oxygen species

\section{Introduction}

Traumatic brain injuries (TBIs) represent a significant socioeconomic burden (Marshall, 2000). In the United States, TBI incidence is 618 per 100,000 person-years (Sosin et al., 1996), and an estimated 80,000-90,000 individuals sustain long-term disabilities annually (Thurman et al., 1999). TBI can result in neurological impairment because of immediate CNS tissue disruption (primary injury), but, additionally, surviving cells may be secondarily damaged by complex mechanisms triggered by the primary event, leading to further damage and disability (Teasdale and Graham, 1998; Amar and Levy, 1999). Several mechanisms have been implicated, including necrotic and apoptotic pathways (for review, see Beattie et al., 2000; Raghupathi et al., 2000; Zipfel et al., 2000). However, their complex interactions have made it difficult to determine which pathways should be addressed therapeutically in TBI. To date, most approaches to the treatment of

Received May 1, 2006; revised Aug. 13, 2006; accepted Sept. 17, 2006.

M.T. was supported by Canadian Institutes of Health Research (CIHR) Grant MOP150807, National Institutes of Health Grant NS048956, and the Canadian Stroke Network. M.T. is a CIHR Clinician Scientist. A.L. is the recipient of a CIHR Doctoral Research Award. H.-S.S. is the recipient of a HSFC Focus on Stroke Postdoctoral Fellowship.

Correspondence should be addressed to Dr. Michael Tymianski, Fellow of the Royal College of Surgeons of Canada, Toronto Western Hospital, WW 4-435, 399 Bathurst Street, Toronto, Ontario, Canada M5T 258. E-mail: mike.tymianski@uhn.on.ca.

DOI:10.1523/JNEUROSCI.3507-06.2006

Copyright $\odot 2006$ Society for Neuroscience $\quad$ 0270-6474/06/2611540-14\$15.00/0
TBI that target a single injury mechanism have failed in clinical trials (Ikonomidou and Turski, 2002; Tolias and Bullock, 2004; McKee et al., 2005). Consequently, it has been suggested that "recognition of multiple cell death pathways should lead to new treatment strategies, including both combination drug treatment and drugs that affect multiple components of the secondary injury cascade" (Faden, 2002). However, compelling evidence to indicate which of the many components of secondary injury should be addressed by these means is lacking. This report begins to address this need.

We previously developed and characterized an in vitro model of neuronal stretch injury to study secondary injury mechanisms (Arundine et al., 2003, 2004). In brief, cultured cortical neurons were exposed to sublethal stretch to remove the confounding effects of primary mechanical damage. The neurons maintained cell membrane integrity, viability, and electrophysiological function but exhibited an enhanced vulnerability to subsequent challenges with L-glutamate or NMDA (Arundine et al., 2003). This was attributable to a combination of stretch-induced, mitochondrially derived, reactive oxygen species (ROS) compounded by NMDA-mediated nitric oxide (NO) production. Together, these formed the lethal oxidant peroxynitrite $(\mathrm{PN})$, which killed the cells (Arundine et al., 2004). However, cell death in the stretch plus NMDA paradigm was also associated with nuclear irregularities and DNA fragmentation suggestive of apoptosis. Because 
apoptotic mechanisms have been implicated in TBI previously (see reviews above), we set out to determine how they interact with PN-dependent neurotoxic mechanisms.

We studied these mechanisms in vitro using a cell stretch paradigm and in vivo using the rodent lateral fluid percussion injury (FPI) model of TBI. We found that neuronal stretch injury induces cytochrome $c$ release into cytoplasm as anticipated by others (Buki et al., 2000; Fiskum, 2000). However, whereas cytochrome $c$ release is expected to trigger classical, caspase-mediated apoptosis (Kluck et al., 1997; P. Li et al., 1997), this process was aborted in traumatized neurons because of the actions of PN. Here, we show that PN inactivates classical apoptosis in vitro and in vivo by directly and preferentially targeting caspase-3, a key effector caspase. This inhibition was primarily attributable to cysteinyl oxidation of caspase- 3 by $\mathrm{PN}$, thus aborting caspasemediated effects and switching cell death away from a classical apoptotic mechanism.

\section{Materials and Methods}

Unless otherwise indicated, all chemicals are from Sigma-Aldrich (Oakville, Ontario, Canada). All in vitro experiments were performed at $37^{\circ} \mathrm{C}$. All experiments were approved by the University Health Network Animal Resource Center, which is fully accredited by the Canadian Council on Animal Care.

Primary murine cortical cultures. Mixed cortical cell cultures containing both neurons and glia were prepared from embryonic day 15 (E15) Swiss mice as described previously (Arundine et al., 2004). In brief, cerebral cortices were extracted from embryos and incubated for $10 \mathrm{~min}$ in $0.05 \%$ trypsin-EDTA. Digested cortices were dissociated by trituration and plated on poly-L-ornithine-coated six-well plates at $\sim 3.6 \times 10^{6}$ cells per well. Flexible membrane plates (Flexcell International Corporation, Hillsborough, NC) were used for stretch injury experiments, and standard plates (Corning, Corning, NY) were used for all other experiments. All cell culture reagents were obtained from Invitrogen (Mississauga, Ontario, Canada). Plating medium consisted of minimum essential medium supplemented with $10 \%$ heat-inactivated horse serum, $10 \%$ fetal bovine serum, $1 \%$ glutamine, and $25 \mathrm{~mm}$ glucose. After $3 \mathrm{~d}$ in vitro, growth of non-neuronal cells was halted by exposure to $10 \mu \mathrm{M}$ FDUsolution ( $5 \mu \mathrm{M}$ uridine, $5 \mu \mathrm{M}(+)$-5-fluor-2'-deoxyuridine) for $48 \mathrm{~h}$. Previous studies have shown that these cultures are $>85 \%$ neurons (Sattler et al., 1997). Cultures were maintained at $37^{\circ} \mathrm{C}$ in a humidified $5 \%$ $\mathrm{CO}_{2}$ atmosphere until used at $11 \mathrm{~d}$ after plating.

3-Morpholinosydnonimine and staurosporine treatments. At $11 \mathrm{~d}$ after dissection, the growth medium is aspirated and cultures are washed twice with HEPES solution. A $100 \mathrm{~mm}$ 3-morpholinosydnonimine (SIN-1) stock was prepared in $\mathrm{ddH}_{2} \mathrm{O}$ immediately before use, and a $100 \mu \mathrm{M}$ staurosporine stock solution was dissolved in DMSO. Appropriate volumes of the stock solution (according the experimental protocol) were added directly to the wells. For the cotreatment paradigm, $10 \mu \mathrm{l}$ of 100 $\mathrm{mm}$ SIN-1 and $10 \mu \mathrm{l}$ of $100 \mu \mathrm{m}$ staurosporine were added directly to the wells, resulting in a final concentration of $1 \mathrm{~mm}$ SIN- 1 and $1 \mu \mathrm{M}$ staurosporine. Accordingly, the vehicle controls for the cotreatment paradigm were treated with $10 \mu \mathrm{l}$ each of $\mathrm{ddH}_{2} \mathrm{O}$ and DMSO. Cultures were gently shaken to promote equal distribution. Treatments were left in the wells until appropriate time points in a $37^{\circ} \mathrm{C}$ humidified incubator for the remainder of the experiments.

In vitro cell stretch model. This was performed as described previously (Arundine et al., 2003, 2004). In brief, the cultures were washed twice with HEPES solution composed of the following (in $\mathrm{mM}$ ): $121 \mathrm{NaCl}, 10$ HEPES acid, 7 HEPES Na, 20 D-glucose, 0.01 glycine, $0.5 \mathrm{KCl}, 1 \mathrm{Na}$ pyruvate, $1.8 \mathrm{CaCl}_{2}$, and $0.3 \mathrm{NaHCO}_{3}$. Cultures were then stretched to $130 \%$ of their original length for $1 \mathrm{~s}$ using the Flexercell FX-3000 Strain Unit (Flexcell, Hillsborough, NC). This degree of stretch did not cause the cultures to detach from their underlying substrate and, in itself, is nonlethal to the cells (Arundine et al., 2004). Immediately after stretch, the cells were washed with either HEPES solution alone or in combination with NMDA for $1 \mathrm{~h}$ and then returned to HEPES solution. Control cultures were identically manipulated, except that no stretch was applied. All solutions contained nimodipine $(2 \mu \mathrm{M})$ and CNQX $(10 \mu \mathrm{M})$ to restrict the actions of applied NMDA to NMDA receptors (NMDARs) by preventing the secondary activation of other pathways (Sattler et al., 1998).

Lateral FPI. FPI was performed as described by McIntosh et al. (1989) using 350-370 g male Sprague Dawley rats (Charles River, St. Constant, Quebec, Canada). In brief, $24 \mathrm{~h}$ before injury, the animal was anesthetized using $2 \%$ halothane and 2:1 nitrous oxide:oxygen. A $4.8 \mathrm{~mm}$ craniotomy was made with its center between the bregma and lambda and between the sagittal suture and right temporal ridge. A modified Leur-loc fitting was attached over the craniotomy and cemented in place using Acron MC/R dental acrylic (GC America, Alsip, IL). The Leur-loc was filled with saline, and a small piece of gelfoam was inserted. The incision was sutured, and the animal was returned to its cage overnight. The following day, the incision was reopened, cleaned, and the gelfoam was removed. The animal was attached to the fluid percussion injury device (Custom Design and Fabrication, Richmond, VA) and was administered a $2.4 \mathrm{~atm}$ injury, which resulted in a mortality rate of $\sim 6 \%$. Injury severity was recorded using an oscilloscope attached to a transducer. Immediately after injury, the Leur-loc setup was removed en bloc, the wound was cleaned, and the incision was resutured. The animal was then returned to its cage.

2,3,5-Triphenyltetrazolium chloride staining. Twenty-four hours after fluid percussion injury, animals were reanesthetized as above and decapitated. Whole brains were extracted and chilled at $-20^{\circ} \mathrm{C}$ for $10 \mathrm{~min}$. Coronal brain sections were made and incubated in $2 \% 2,3,5$ triphenyltetrazolium chloride (TTC) in saline solution for $30 \mathrm{~min}$ at $37^{\circ} \mathrm{C}$ (Joshi et al., 2004).

Protein extraction. After in vitro treatments, cells were washed twice with HEPES solution. Mammalian protein extraction reagent (M-PER; Pierce, Rockford, IL) was added to each well, and the cell lysates were collected and centrifuged at 10,000 rpm for $10 \mathrm{~min}$. Supernatant was collected for analysis in activity assays and Western blots. After FPI, brain tissue samples were extracted from TTC-treated brains and placed into modified radioimmunoprecipitation assay buffer (50 mM Tris- $\mathrm{HCl}, 150$ mм NaCl, 1 mм EDTA, pH 7.4) with protease inhibitors for Western blotting. Protease inhibitors were omitted for caspase activity assays. To extract protein from the injury site, all cortical areas negatively stained with TTC were extracted from $2 \mathrm{~mm}$ sections spanning from approximately -2.0 to $-9.0 \mathrm{~mm}$ (bregma levels) and combined per animal for analysis. Brain sections were triturated using a 25-gauge needle, followed by finer dissociation using a 30 -gauge needle. Samples were centrifuged at $2900 \times g$ for $5 \mathrm{~min}$, and pellets were discarded. Samples were stored at $-80^{\circ} \mathrm{C}$ until used. Protein concentrations from both extractions were determined using the Bio-Rad Protein Assay (Bio-Rad, Hercules, CA) in conjunction with bovine serum albumin standards.

SDS-PAGE. Each lane was loaded with $20 \mu \mathrm{g}$ of boiled sample suspended in $4 \times$ sample buffer consisting of the following: $0.125 \mathrm{M}$ Tris$\mathrm{HCl}, \mathrm{pH} 6.8,20 \%$ glycerol, $4 \%$ SDS, $5 \% \beta$-mercaptoethanol, and $0.025 \%$ bromophenol blue. Gels were run at $70 \mathrm{~V}$. All Western blots were run on 12\% Tris/glycine gels except poly-(ADP-ribose) polymerase-1 (PARP-1) gels, which were run with $7.5 \%$ Tris/glycine gels. Gels were transferred onto nitrocellulose paper in $4^{\circ} \mathrm{C}$ in transfer buffer $(0.2 \mathrm{M}$ glycine, $0.025 \mathrm{M}$ Tris, $0.04 \%$ SDS, $20 \%$ methanol) at $100 \mathrm{~V}$ for $1 \mathrm{~h}$.

Immunoblotting. After transfer, gels were blocked in $5 \%$ blotting grade blocker nonfat dry milk (Bio-Rad) in TBST $(0.01 \mathrm{~m}$ Tris, $0.1 \mathrm{M} \mathrm{NaCl}$, $0.05 \%$ Tween 20 ) for $50 \mathrm{~min}$. Immunoblots were performed with either a monoclonal mouse anti-cytochrome $c$ antibody (1:200; PharMingen, San Diego, CA); polyclonal rabbit anti-caspase-3 antibody (1:1000; Stressgen, Victoria, British Columbia, Canada) recognizing both active and inactive (pro-) caspase-3; polyclonal rabbit anti-3-nitrotyrosine antibody ( $2 \mu \mathrm{g} / \mathrm{ml}$; Upstate Biotechnology, Lake Placid, NY); monoclonal mouse anti-3-nitrotyrosine ( $2 \mu \mathrm{g} / \mathrm{ml}$; Biomol, Plymouth Meeting, PA); polyclonal rabbit anti-caspase-9 (1:500; Stressgen); monoclonal mouse anti-apoptotic protease activating factor-1 (APAF-1) (1:500; Stressgen); monoclonal anti-cytochrome $c$ (1:200, PharMingen); or monoclonal mouse anti-PARP-1 (2 $\mu \mathrm{g} / \mathrm{ml}$; PharMingen) recognizing both cleaved and uncleaved PARP-1. Primary antibodies were incubated overnight at 
$4^{\circ} \mathrm{C}$. After washing, HRP-conjugated goat anti-rabbit IgG (1:5000; Jackson ImmunoResearch, West Grove, PA) or HRP-conjugated goat antimouse IgG (1:5000; Pierce Biotechnology) was added at room temperature for $1 \mathrm{~h}$. Immunoreactivity was visualized using an ECL Western blotting detection system (GE Healthcare, Piscataway, NJ). To ensure equal protein loading in all lanes within each immunoblot, the amount of protein pipetted into each lane (usually $40 \mu \mathrm{g}$ ) was quantified using the Bio-Rad Protein Assay Dye Reagent (catalog \#500-0006). Also, the blots were then stripped and reprobed with anti- $\beta$-actin antibodies (1:5000; Sigma). As a further confirmation, proteins from the same sample lysates were run on parallel gels, transferred onto nitrocellulose, and stained with Ponceau stain. Representative examples are provided in Figure $2 \mathrm{~A}$ but are generally omitted for clarity.

Subcellular fractionation. HEPES solution in the wells was aspirated and replaced with $1 \mathrm{ml}$ of ice-cold PBS. Cells were collected and samples were centrifuged at $300 \times g$ for $1 \mathrm{~min}$ at $4^{\circ} \mathrm{C}$. The supernatant was discarded, and the pellet was resuspended in $300 \mu \mathrm{l}$ of ice-cold PBS. Samples were centrifuged at $300 \times g$ for $1 \mathrm{~min}$ at $4^{\circ} \mathrm{C}$ and supernatant was discarded. The pellet was then resuspended in $500 \mu$ l of digitonin release buffer (250 mm sucrose, $17 \mathrm{~mm}$ 4-morpholinepropanesulfonic acid, 2.5 $\mathrm{mm}$ EDTA, $0.8 \mathrm{mg} / \mathrm{ml}$ digitonin) and transferred to a $2 \mathrm{ml}$ Dounce homogenizer. Samples were homogenized with 30 ups and downs. Lysates were spun at $5000 \times g$ for $5 \mathrm{~min}$, the supernatant was discarded, and the lysates were centrifuged again at $21,000 \times g$ for $30 \mathrm{~min}$ all at $4^{\circ} \mathrm{C}$. The supernatant was collected and designated the cytoplasmic fraction, whereas the pellet was resuspended in $250 \mu \mathrm{l}$ of M-PER and designated the mitochondrial fraction.

Terminal deoxynucleotidyl transferase-mediated biotinylated UTP nick end labeling assay. Nuclear DNA strand breaks were detected by enzymatically labeling free $3^{\prime}-\mathrm{OH}$ termini with modified nucleotides provided in the Apoptag kit (Intergen, Purchase, NY) using the manufacturer instructions. Visualization of terminal deoxynucleotidyl transferasemediated biotinylated UTP nick end labeling (TUNEL)-stained nuclei was achieved using an anti-digoxigenin antibody conjugated to a fluorescein or peroxidase reporter molecule (ApopTag Kits S7110 and S7100, respectively). In fluorescence experiments, nuclei were counterstained using $5 \mathrm{ng} / \mathrm{ml}$ Hoechst 33258 (Invitrogen, Eugene, OR) and visualized using 465-495 nm excitation and 515-555 nm emission for fluorescein and $340 \mathrm{~nm}$ excitation and $510-40 \mathrm{~nm}$ emission for Hoechst. The number of TUNEL-positive cells was expressed as a fraction of the total cell number in the field.

Phosphatidylserine inversion. After chemical treatment, the cells were washed twice with HEPES solution. A kit using FITC-conjugated annexin $\mathrm{V}$ to label inverted phosphatidylserine (ApopNexin kit; Intergen) was used according to the specifications of the manufacturer. After application of the labeled annexin $\mathrm{V}$, the cells were washed with HEPES solution and observed at $200 \times$ magnification and visualized using 465 $495 \mathrm{~nm}$ excitation and 515-555 nm emission for fluorescein.

Quantification of in vitro cell death. Cell death was determined by serial quantitative measurements of propidium iodide (PI) fluorescence using a multiwell plate fluorescence scanner (Fluoroskan Ascent FL; ThermoLab Systems, Burlington, Ontario, Canada) as described previously (Sattler et al., 1997, 1998). In brief, the medium in each culture well was replaced with HEPES solution containing $50 \mu \mathrm{g} / \mathrm{ml} \mathrm{PI}$, and a baseline fluorescence reading was taken. Sequential readings were then taken at appropriate intervals over the observation period. The fraction of dead cells in each culture at a given time was calculated as: fraction dead $=$ $\left(F_{\mathrm{t}}-F_{\mathrm{o}}\right) / F_{\mathrm{NMDA}}$ where $F_{\mathrm{t}}$ is the PI fluorescence at time $t, F_{\mathrm{o}}$ is the initial PI fluorescence at time 0 , and $F_{\mathrm{NMDA}}$ is the background subtracted PI fluorescence of identical cultures from the same dissection and plating, $20-24 \mathrm{~h}$ after a $60 \mathrm{~min}$ exposure to $1 \mathrm{~mm} \mathrm{NMDA}$ at $37^{\circ} \mathrm{C}$. Based on manual observations at the time of validation of this technique, this NMDA exposure routinely produced near complete neuronal death in each culture but had no effect on surrounding glia (Bruno et al., 1994; David et al., 1996; Sattler et al., 1997). Adding Triton X-100 (0.1\%) to cultures treated in this manner produced an additional $10-15 \%$ increase in PI fluorescence resulting from permeabilization of non-neuronal cell membranes, consistent with a $10-15 \%$ glial component in the cultures.

Fluorogenic quantification of caspase-3 and caspase-9 activity. After quantification of protein concentration, cell lysates from in vitro experiments were incubated with $10 \mu \mathrm{M} \mathrm{N}$-acetyl-Asp-Glu-Val-Asp-7-amino4-methylcoumarin (EMD Biosciences, San Diego, CA) in M-PER at $37^{\circ} \mathrm{C}$ for $1 \mathrm{~h}$. Protein lysates from whole animal experiments were treated identically, except $40 \mu \mathrm{M}$ Ac-DEVD-AMC was used for caspase- 3 activity measurements, and $100 \mu \mathrm{M}$ Ac- $N$-acetyl-Leu-Glu-His-Asp-7-amidotrifluoromethylcoumarin (EMD Biosciences) was used to measure caspase- 9 activity. Plates were placed into a multiwell plate fluorescence scanner, and fluorescence was quantified at $355 \mathrm{~nm}$ excitation and 460 $\mathrm{nm}$ emission for Ac-DEVD-AMC, or $380 \mathrm{~nm}$ excitation and $510 \mathrm{~nm}$ emission for Ac-LEHD-AFC. We note here that these fluorogenic caspase substrates have the highest specificity to caspase- 3 and caspase- 9 , respectively, but we do not rule out possible interactions with other effector or initiator caspases, respectively. However, any additional, as yet undemonstrated, interactions with these caspases would not affect the interpretation of the results provided herein.

Ex vivo recombinant caspase-3 activity system. Recombinant procaspase-3 was resuspended in PBS, $\mathrm{pH} 7.4$, to a concentration of $0.5 \mu \mathrm{g} / \mathrm{ml}$ and incubated with SIN-1 or peroxynitrite (EMD Biosciences) for $1 \mathrm{~h}$ at $37^{\circ} \mathrm{C}$. In some experiments, $10 \mathrm{~mm}$ dithiothreitol (DTT) or $\mathrm{ddH}_{2} \mathrm{O}$ as a negative control was added to the system. To trigger caspase- 3 activity, $0.5 \mathrm{ng}$ ( $3 \mathrm{U}$ ) of recombinant caspase-9 (Calbiochem, La Jolla, CA) was added. Activity was assessed using Ac-DEVD-AMC as above.

Ex vivo recombinant caspase-9 activity system. Recombinant caspase-9 (Calbiochem; $1 \mathrm{U}$ ) was diluted in M-PER to a final concentration of 0.01 $\mathrm{U} / \mu \mathrm{l}$ and incubated with peroxynitrite for $1 \mathrm{~h}$ at $37^{\circ} \mathrm{C}$. DTT $(10 \mathrm{~mm})$, or $\mathrm{dd}_{2} \mathrm{O}$ as a negative control, was applied immediately after incubation with peroxynitrite. Caspase- 9 activity was assessed using Ac-LEHD-AFC as above.

Immunoprecipitation. Protein lysates from cell cultures were incubated with agarose beads and agarose beads conjugated to nonspecific mouse IgG before incubation with agarose-conjugated anti-3-nitrotyrosine (Upstate Biotechnology). Lysates were incubated overnight at $4^{\circ} \mathrm{C}$ with agarose-conjugated anti-3-nitrotyrosine, and the supernatant was discarded. The beads were suspended in $2 \times$ sample buffer without $\beta$-mercaptoethanol and boiled for $10 \mathrm{~min}$ before being run with SDSPAGE. Gels were then transferred for Western blotting.

DNA gel electrophoresis. At $20 \mathrm{~h}$ after the experimental manipulation, cells were collected from each tissue culture well in HEPES solution and pelleted by centrifugation at $1000 \mathrm{rpm}$. The cells were digested for $2 \mathrm{~h}$ at $56^{\circ} \mathrm{C}$ in DNA lysis buffer containing the following (in mM): 10 Tris, $\mathrm{pH}$ 8.0, 2 EDTA, pH 8.0, $400 \mathrm{NaCl}, 0.5 \%$ SDS, and $1 \mathrm{mg} / \mathrm{ml}$ proteinase $\mathrm{K}$. Total DNA was subsequently extracted and precipitated using the phenol/chloroform method. In brief, an equal volume of phenol was added to the cell extract, followed by centrifugation at 13,000 rpm for $10 \mathrm{~min}$. The top layer was removed, and an equal volume of chloroform was added thereafter. This process was repeated twice. After the final chloroform extraction, $100 \%$ ethanol was added to the top fraction and placed on ice for $10 \mathrm{~min}$. DNA was pelleted by centrifugation 13,000 rpm for 10 min. The DNA pellet was dried, and $50 \mu \mathrm{l}$ of $\mathrm{dd}_{2} \mathrm{O}$ was added thereafter. The DNA pellet was left to dissolve into the $\mathrm{ddH}_{2} \mathrm{O}$ overnight at $4^{\circ} \mathrm{C}$, gently mixed, and then quantified spectrophotometrically (260/280 absorption). DNA $(10 \mu \mathrm{g})$ was gently mixed with loading buffer, loaded on a $2 \%$ agarose gel, and electrophoretically separated at $100 \mathrm{~V}$. Gels were stained with ethidium bromide and photographed using UV transillumination.

Immunohistochemistry. For frozen brain sections, animals were anesthetized and transcardially perfused using heparinized saline followed by $4 \%(\mathrm{w} / \mathrm{v})$ paraformaldehyde in PBS. Whole brains were extracted from the fixed animals and were cryoprotected in 30\% sucrose with $4 \%$ paraformaldehyde in PBS solution at $4^{\circ} \mathrm{C}$ overnight. They were sectioned into $25 \mu \mathrm{m}$ free-floating slices in PBS using a Kryostat 1720 (Leica, Wetzler, Germany). Immunostaining was performed as described previously (Sun et al., 2006). In brief, sections were blocked using a 1\% BSA, 3\% goat serum, $0.3 \%$ Triton X-100 in PBS solution for $90 \mathrm{~min}$ and doubled labeled with rabbit anti-3-nitrotyrosine (1:50; Upstate Biotechnology) and mouse anti-neuronal-specific nuclear protein (NeuN; 1:100; Chemicon, Temecula, CA) overnight at $4^{\circ} \mathrm{C}$. Sections were subsequently washed and blocked briefly with blocking solution and incubated with goat anti-rabbit Alexa568 (1:100; Invitrogen) and goat anti-mouse FITC 
A

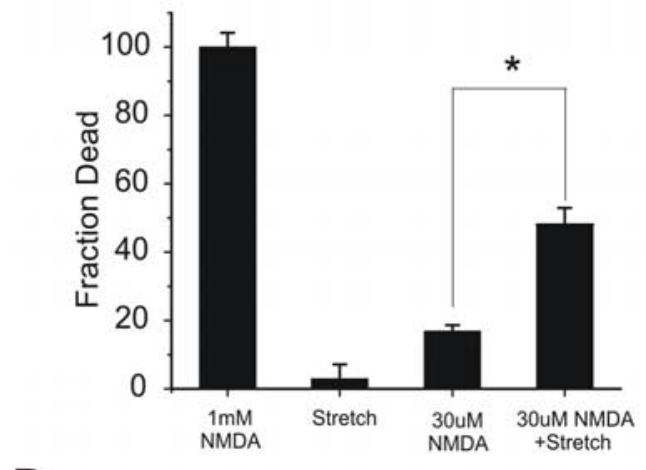

B

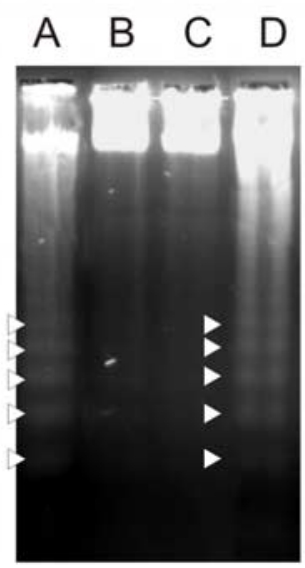

B: Control

C: Stretch

D: Stretch
C
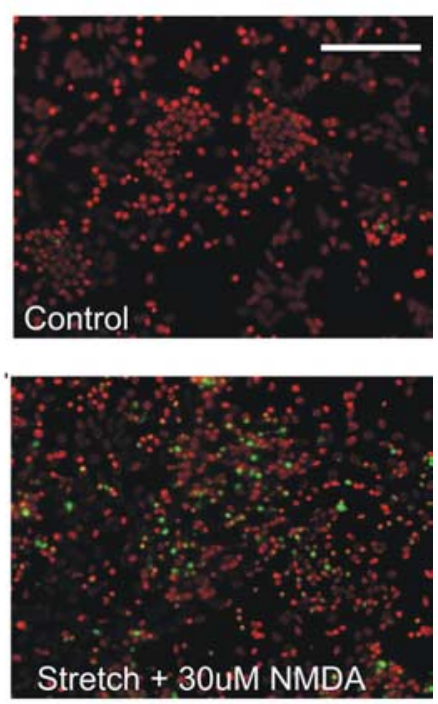
30uM NMDA

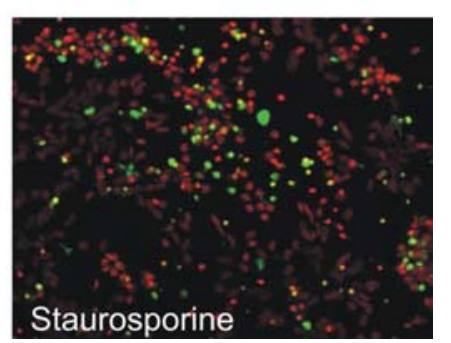

Figure 1. Enhanced vulnerability and apoptosis-like features of sublethally stretched neurons in response to NMDA. $\boldsymbol{A}$, Neuronal death $20 \mathrm{~h}$ after the indicated insults as assessed by propidium iodide uptake. Where used, NMDA was applied for $1 \mathrm{~h}$ after the stretch. Asterisk, Difference from $30 \mu \mathrm{MNMDA}\left({ }^{*} p<0.05 ; n=4\right)$. $\boldsymbol{B}$, Staurosporine and stretch plus $30 \mu \mathrm{m} \mathrm{NMDA}$, but not stretch alone, induces internucleosomal DNA laddering (arrowheads) after insult. Data are representative of three separate experiments. C, Staurosporine and stretch plus $30 \mu \mathrm{m}$ NMDA, but not HEPES-treated controls, enhances TUNEL (green) labeling of the cultures. Cultures were costained with Hoescht 33258 to illustrate cell nuclei (red). Images are representative of three separate experiments. Scale bar, $200 \mu \mathrm{m}$.

(1:200; Sigma) for $1 \mathrm{~h}$ at room temperature. Slides were mounted using ProLong Gold antifade reagent (Invitrogen).

For paraffin-embedded tissue, anesthetized animals were transcardially perfused using $10 \%$ phosphate-buffered formalin and decapitated, and the heads were placed into $10 \%$ phosphate-buffered formalin overnight. Whole brains were extracted the following day, processed, embedded in paraffin wax, and sectioned into $7-\mu \mathrm{m}$-thick slices. The slices were kept at room temperature until use, after which they were rehydrated in a reverse order of decreasing xylene and alcohol concentrations. TUNEL staining was performed according to the manufacturer protocol using an Apoptag kit (Intergen). After TUNEL staining, the specimens were washed with PBS and blocked using 1\% BSA in PBS for $30 \mathrm{~min}$. A secondary block with $2 \%$ goat serum in PBS for $1 \mathrm{~h}$ was followed by treatment with 1:100 anti-3-nitrotyrosine (Upstate Biotechnology) overnight at $4^{\circ} \mathrm{C}$. The next morning, sections were treated with anti-rabbit FITC (1:1000; Jackson ImmunoResearch) for $1.5 \mathrm{~h}$.

Confocal microscopy. Frozen sections $(25 \mu \mathrm{m})$ taken from brains of animals that underwent a $2.4 \mathrm{~atm}$ FPI were double stained with antiNeuN and anti-3-nitrotyrosine. These were visualized with a laserscanning confocal microscope (Zeiss LSM 510 META) using $25 \times$ or $63 \times$ microscope lenses (Zeiss). In each preparation, the section was initially scanned to determine a depth halfway between its two surfaces, and the midslice depth was used for imaging. Optical stacks of 5-10 confocal images taken at $0.5 \mu \mathrm{m}$ intervals were used to generate figures as described previously (Sun et al., 2006).

Statistics. All data were expressed as mean \pm SEM and analyzed using ANOVA or Student's $t$ test as appropriate (SigmaStat3.0; SPSS, Chicago, IL). Statistical significance was considered at the $p<0.05$ level.

\section{Results}

Sublethally stretched neurons exhibit enhanced vulnerability to NMDA toxicity and apoptotic-like DNA fragmentation

We previously developed and characterized a model of sublethal neuronal stretch injury to study secondary mechanisms of traumatic damage (see Materials and Methods). In brief, stretched neurons maintain viability, cell membrane integrity, and electrophysiological function but exhibit a heightened vulnerability to subsequent challenges with NMDAR agonists (Arundine et al., 2003). Consistent with this, cultured cortical neurons subjected to $130 \%$ stretch for $1 \mathrm{~s}$ (hereafter termed "stretch") were unharmed by this insult as measured by PI uptake (Fig. 1A). However, the addition of $30 \mu \mathrm{M}$ NMDA induced significantly more cell death in stretched compared with unstretched cultures. The enhanced vulnerability of stretched neurons to NMDA insults is the consequence of a combination of stretchinduced, mitochondrially derived, ROS compounded by NMDA-mediated NO production. Together, these form the lethal oxidant PN, which accounts for the lethality of the stretch plus NMDA paradigm (Arundine et al., 2004).

Neurons subjected to stretch plus 30 $\mu \mathrm{M}$ NMDA, but not neurons exposed to control medium or to stretch without NMDA, exhibited internucleosomal DNA fragmentation as demonstrable by DNA gel electrophoresis (Fig. 1B). The "DNA ladder" was similar to that obtainable by treating the cultures with staurosporine, a nonselective protein kinase inhibitor, which induces caspase- 3 activation and classical apoptosis in cultured central neurons (Koh et al., 1995). Also, neurons subjected to stretch plus NMDA exhibited increased TUNEL, a feature that was also produced by the staurosporine treatment (Fig. 1C). Thus, stretch plus NMDA injury resulted in cell death exhibiting apoptotic-like features.

\section{Neuronal stretch induces cytoplasmic cytochrome $c$ release but without concomitant caspase-3 activation}

In vitro, neuronal stretch produces mitochondrial dysfunction and ROS formation (Arundine et al., 2004). In vivo, neurotrauma is associated with the release of mitochondrial cytochrome $c$ into cytoplasm (Buki et al., 2000; Fiskum, 2000). Because cytochrome $c$ release is recognized as a trigger of classical type II caspasemediated apoptosis (Kluck et al., 1997; P. Li et al., 1997), we further investigated the cause of DNA degradation in our stretched neurons by measuring cytochrome $c$ release after stretch. Neurons were subjected to the stretch plus NMDA insult, and cytochrome $c$ immunoreactivity in cytoplasmic fractions was determined by Western blots. Stretch plus $30 \mu \mathrm{M}$ NMDA induced a rapid and robust increase in cytoplasmic cytochrome $c$, which was measurable immediately after the insult and persisted for at $\geq 20$ h (Fig. 2A).

Cytochrome $c$ release is the critical point in the formation of 
the apoptosome, a cytoplasmic protein complex that leads to the processing and activation of effector caspases that, in turn, lead to apoptotic neuronal death (for review, see Green and Reed, 1998; Stefanis, 2005). Although apoptosis is not always caspase dependent, it is anticipated that once released, cytochrome $c$ should lead to caspase- 3 activation (Dawson and Dawson, 2004). To test this, whole-cell lysates were taken from cultures subjected to stretch plus NMDA and assayed in Western blots using an anticaspase- 3 antibody recognizing both active (cleaved) and inactive (pro-) caspase-3 (molecular weight of $\sim 17 \mathrm{kDa}$ and $\sim 32 \mathrm{kDa}$, respectively; see Materials and Methods). Surprisingly, no active caspase-3 immunoreactivity was observed at the expected $\sim 17$ $\mathrm{kDa}$ band (Fig. $2 \mathrm{~B}$ ). To probe caspase- 3 proteolytic activity, we incubated the whole-cell lysates taken from cells exposed to stretch plus NMDA with the fluorogenic caspase-3 substrate AcDEVD-AMC (10 $\mu \mathrm{M}$; see Materials and Methods). This yielded no increase in AMC fluorescence $20 \mathrm{~h}$ after the insult when compared with controls (Fig. $2 C$ ). Staurosporine ( $1 \mu \mathrm{M})$, the positive control that elicits caspase-3-mediated classic apoptosis in neurons (Koh et al., 1995), induced both caspase-3 cleavage (Fig. 2 B, right lane) and significantly increased Ac-DEVD-AMC fluorescence (Fig. 2C). Thus, despite robust cytoplasmic cytochrome $c$ release (Fig. $2 \mathrm{~A}$ ), the cleavage and activity of caspase-3, the main

A

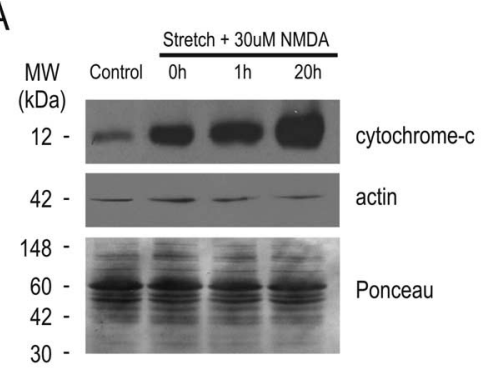

C

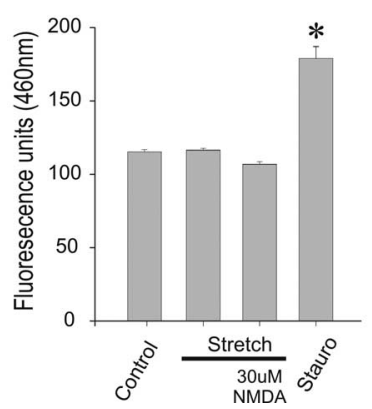

Figure 2. Stretch plus $30 \mathrm{~mm}$ NMDA injury produces cytochrome crelease, but not caspase-3 activation. $\boldsymbol{A}$, Immunoblot of the cytoplasmic fraction of injured cells using anti-cytochrome $\boldsymbol{c}$ (top) antibodies. Reblotting with anti- $\beta$-actin (middle) antibodies and Ponceau staining of parallel samples (bottom) was performed as control for protein loading. Cytochrome $c$ is released into cytoplasm almost immediately following stretch plus $30 \mu \mathrm{m}$ NMDA and persists for at least $20 \mathrm{~h}(n=3)$. $\boldsymbol{B}$, Immunoblot of caspase-3 (both pro- and active/cleaved forms) from whole-cell lysates taken $20 \mathrm{~h}$ after the indicated insult. The procaspase-3 form ( $\sim 32 \mathrm{kDa}$ ) was detectable under all conditions, whereas only cultures treated with staurosporine displayed the active caspase- 3 band $(\sim 17 \mathrm{kDa}$ ). Data are representative of four experiments. C, Cell lysates taken $20 \mathrm{~h}$ after the indicated insults and incubated with Ac-DEVD-AMC show no increases in caspase-3-like proteolytic activity compared with controls ( $n=3$ experiments). Asterisk, Difference from HEPES buffer-treated controls $\left({ }^{*} p<0.05 ; n=3\right)$. Stauro, Staurosporine ( $\left.1 \mu \mathrm{m}\right)$ controls harvested at $24 \mathrm{~h}$ posttreatment in $\boldsymbol{B}$ and $\boldsymbol{C}$. $\boldsymbol{D}$, Immunoblot of whole-cell lysates taken $20 \mathrm{~h}$ after the indicated insults using anti-3-nitrotyrosine antibodies ( $n=4$ experiments). SIN-1 (1 mM for $24 \mathrm{~h}$ ) was used as a positive control. effector caspase of apoptosis, was undetectable in stretch plus NMDA injured neurons.

\section{Neuronal stretch injury is associated with increased peroxynitrite formation}

Cell death in the in vitro stretch model of neuronal injury was associated with PN formation (Arundine et al., 2004), and PN formation has been reported to trigger cell death in neurons and neuronal cell lines (Estevez et al., 1995, 1998; Spear et al., 1998; Virag et al., 1998; Bao and Liu, 2003). Thus, we next examined PN formation in our model by measuring protein tyrosine nitration. Tyrosine nitration occurs nearly exclusively by PN (Beckman and Koppenol, 1996) and is detectable immunologically using anti-3nitrotyrosine antibodies (Ye et al., 1996).

Western blots were performed using whole-cell lysates taken from cultures that had undergone stretch in paradigms identical to those in Figure 2, $B$ and $C$, and then immunostained with an anti-3-nitrotyrosine antibody (see Materials and Methods). Stretch alone failed to elicit nitrotyrosine formation, whereas stretch plus $30 \mu \mathrm{M}$ NMDA elicited increased tyrosine nitration, confirming that PN was formed under this condition (Fig. 2D). SIN-1 (1 mM), a compound known to release equimolar amounts of superoxide and nitric oxide to form peroxynitrite in cortical cultures (Trackey et al., 2001), was used as a positive control for peroxynitrite production and 3-nitrotyrosine detection.

From these findings, we conclude that in vitro neuronal stretch injury elicits a form of cell death characterized by cytochrome $c$ release, PN formation, and DNA fragmentation but

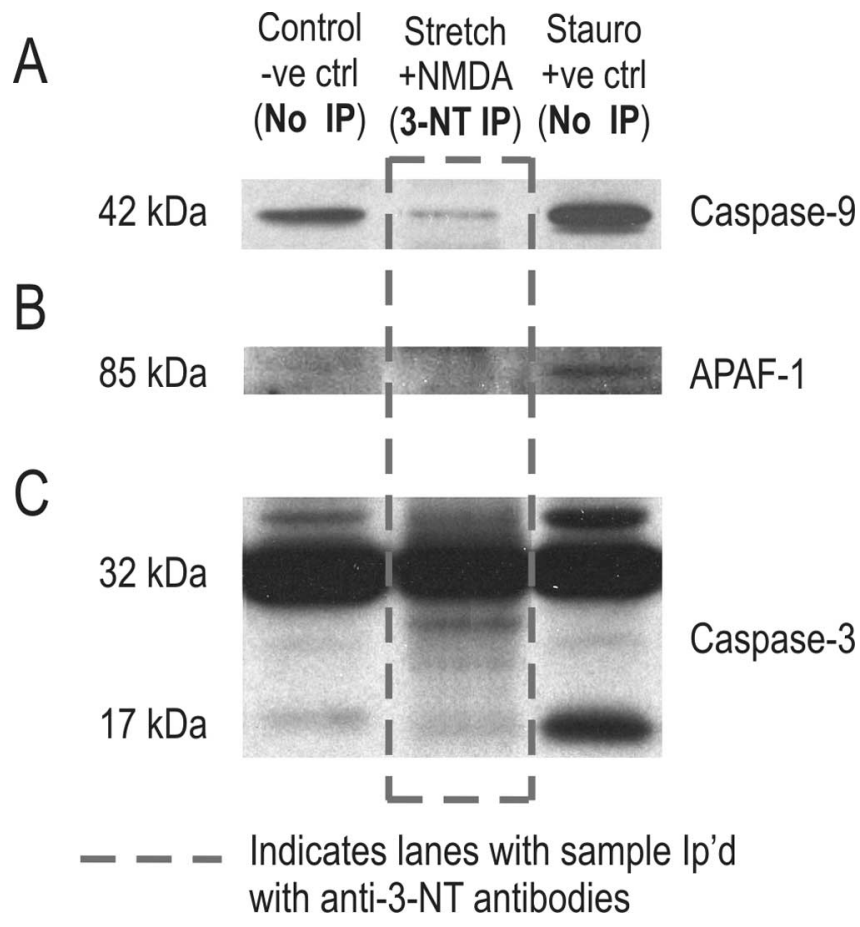

Figure 3. Stretch plus $30 \mu \mathrm{m}$ NMDA nitrates procaspase-3. $\boldsymbol{A}$-C, Whole-cell lysates from cultures exposed to stretch plus $30 \mu \mathrm{m}$ NMDA were immunoprecipitated using an agarosebound monoclonal anti-3-nitrotyrosine antibody and run on immunoblots (middle lanes) along with nonimmunoprecipitated samples from staurosporine-treated cultures as positive controls and from untreated cultures as negative controls. $\boldsymbol{A}$, Anti-caspase- 9 antibody. $\boldsymbol{B}$, Anti-Apaf- 1 antibody. C, Anti-caspase-3 (pro- and active/cleaved forms). $n=2$ experiments/blot. The dotted line encompasses the lanes containing the immunoprecipitated sample. The untreated and staurosporine controls were not immunoprecipitated with anti-3-NT antibodies as, in the absence of nitration, these proteins cannot be precipitated with the antibody-agarose conjugate. 
A

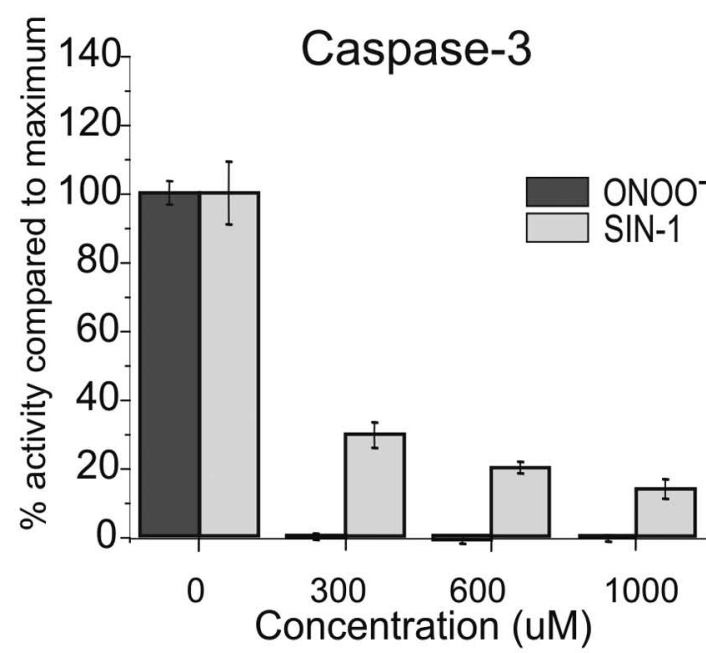

B
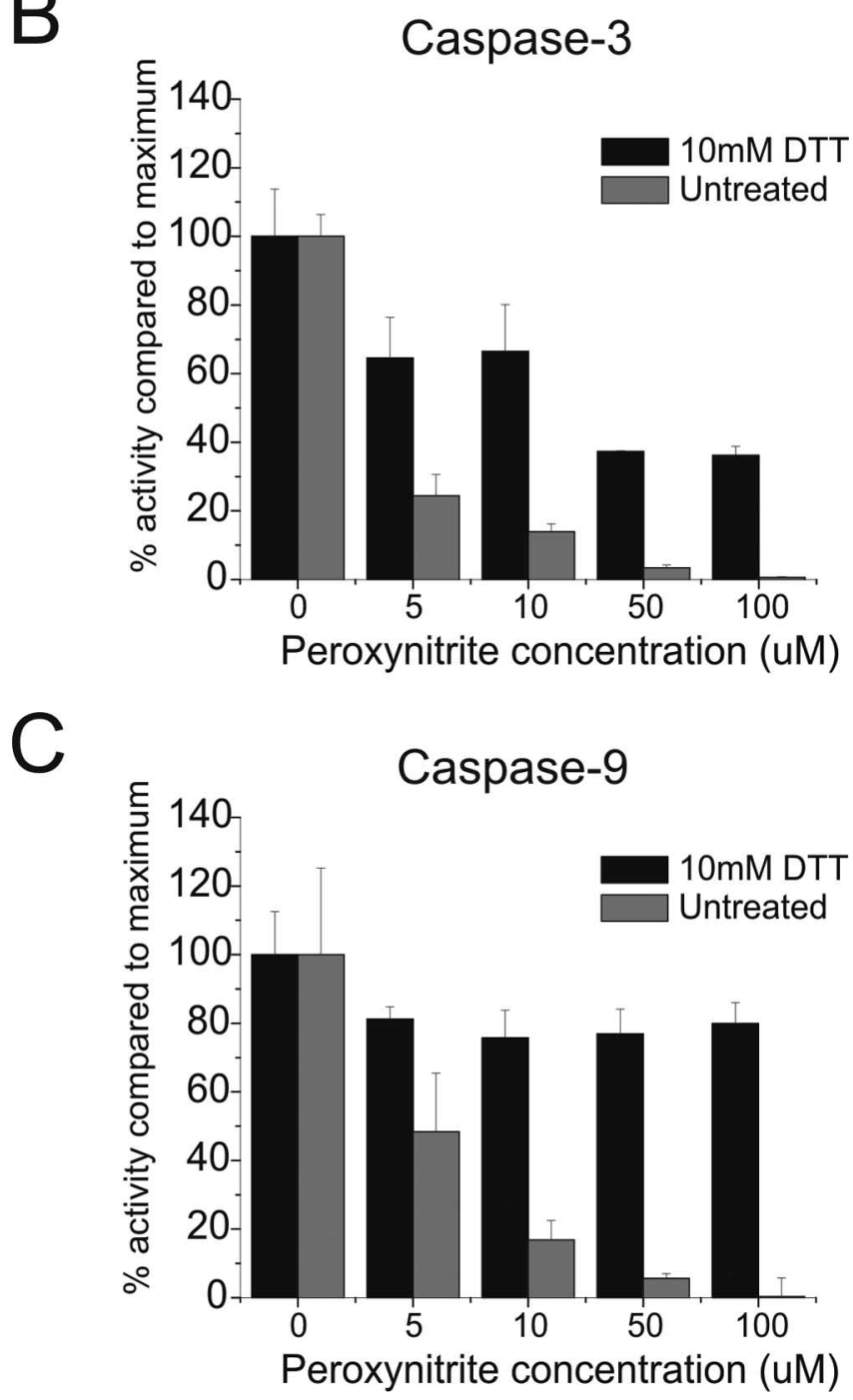

Figure 4. Peroxynitrite can inhibit caspase-3 and caspase-9 activity ex vivo via cysteinyl oxidation. $\boldsymbol{A}$, Effects of the PN donor SIN-1, or of direct PN addition, on caspase-3-like activity. Recombinant procaspase- 3 was incubated with the indicated concentrations of SIN- 1 or peroxynitrite before activation with recombinant caspase- 9 and Ac-DEVD-AMC fluorescence was measured. Both compounds significantly reduced ( $p<0.05)$ caspase-3 activity as shown $(n=$ without caspase-3 activation. Although $\mathrm{PN}$ is known to produce DNA fragmentation without apoptosis (Bolanos et al., 1997; Szabo and Ohshima, 1997; Hill et al., 2000), cytochrome $c$ release is widely viewed as a step that initiates caspase-mediated apoptotic death pathways (Green and Reed, 1998). Thus, to account for the lack of caspase- 3 activation, we hypothesized that, in addition to damaging neurons directly, $\mathrm{PN}$ actively inhibits classical apoptosis in mechanically injured neurons.

Procaspase- 3 is nitrated after stretch injury in cortical neuronal cultures

We next asked whether, in the stretch injury model, PN may act on caspase- 3 directly or whether it acts earlier on steps that intervene between cytochrome $c$ release and caspase- 3 activation. After its release, cytochrome $c$ should bind with procaspase- 9 and Apaf-1 to form an apoptosome complex (P. Li et al., 1997). Apoptosome formation effectively activates caspase-9 (Zou et al., 1999), leading to the cleavage and activation of caspase-3. However, treatment of cytochrome $c$ with peroxynitrite did not affect apoptosome formation and subsequent caspase- 3 activation in previous studies (Ueta et al., 2003). Consequently, we evaluated the interaction of PN with key constituents downstream from cytochrome $c$ by measuring tyrosine nitration of caspase-9, Apaf-1, and caspase- 3 from stretched neurons. Nitrated proteins taken from cortical cultures subjected to stretch plus $30 \mu \mathrm{M}$ NMDA were immunoprecipitated by incubating whole-cell lysates with an anti-3-nitrotyrosine agarose conjugate (see Materials and Methods). The immunoprecipitated lysates were then run on SDS-PAGE and assayed with appropriate antibodies. As controls, we used untreated cultures (negative control) and cultures treated with staurosporine ( $1 \mu \mathrm{m}$; positive control for identification of the immunoreactive bands). Whereas caspase-9 (Fig. 3A) and Apaf-1 (Fig. $3 B$ ) were detectable in the nonimmunoprecipitated positive (staurosporine) controls, neither could be immunoprecipitated with anti-3-nitrotyrosine antibodies, arguing against protein nitration of these apoptosome components after stretch injury. However, the same immunoprecipitates exhibited a strong immunoreactive band at $\sim 32 \mathrm{kDa}$ corresponding to the pro- (inactive) form of caspase-3 (Fig. 3C), indicating that procaspase- 3 is directly nitrated by $\mathrm{PN}$ after the stretch injury

\section{Peroxynitrite inhibits caspase activity directly via cysteine oxidation}

Tyrosine nitration is an index of PN activity but may not necessarily lead to caspase-3 inactivation. Having shown that PN elicits the tyrosine nitration of procaspase-3 (Fig. 3C), we next used an ex vivo system to measure the effects of $\mathrm{PN}$ on the activity of both caspase- 3 and -9 .

First, to examine the direct effect of PN on caspase- 3 activity, recombinant procaspase- 3 was reconstituted in $\mathrm{PBS}, \mathrm{pH} 7.4$, at $0.5 \mu \mathrm{g} / \mathrm{ml}$ and incubated with either SIN-1 or pure peroxynitrite (Fig. $4 A$ ). After $1 \mathrm{~h}$ incubation at $37^{\circ} \mathrm{C}$, recombinant active caspase- 9 (Sigma) was added to the system, and caspase- 3 activity was assessed using the fluorogenic Ac-DEVD-AMC assay (see Materials and Methods). Caspase- 3 activity was significantly decreased after treatment with as little as $300 \mu \mathrm{M}$ SIN-1 and was $\leftarrow$

3 experiments per compound; asterisks, different from own control). $\boldsymbol{B}, \boldsymbol{C}$, Recombinant caspase-3 $(\boldsymbol{B})$ and caspase-9 $(\boldsymbol{C})$ were incubated for $1 \mathrm{~h}$ with the indicated PN concentrations, and proteolytic activity was assessed using Ac-DEVD-AMC and Ac-LEHD-AFC, respectively. The inhibition of activity of both caspases by PN was significantly reversed by DTT (10 mm; $p<0.05$; $n=3$ experiments per concentration/condition). 
completely abolished with peroxynitrite treatment at a similar concentration.

$\mathrm{PN}$ may affect protein function by protein tyrosine nitration (Ischiropoulos et al., 1992; Beckman and Koppenol, 1996) but is also a potent oxidant that affects cysteine residues and may thus exert its effects in part by oxidizing tissue sulfhydryls (Radi et al., 1991). Although PN elicits the tyrosine nitration of procaspase-3 (Fig. $3 C$ ), we have not yet determined whether this posttranslational modification, or cysteine oxidation, is the mechanism of caspase inhibition. Consequently, we next repeated the same ex vivo experiment using a lower range of $\mathrm{PN}$ concentrations $(0-100 \mu \mathrm{M})$. Then, after $1 \mathrm{~h}$ of incubation, we added 10 mm DTT, a sulfhydryl reducing reagent. This treatment significantly reduced the inhibition of caspase- 3 activity by $\mathrm{PN}$, indicating that an oxidative modification of cysteine residues is primarily responsible for the inhibition of caspase- 3 in this recombinant system (Fig. $4 B)$.

Last, to test whether PN can have similar effects on caspase-9, recombinant caspase-9 was incubated with $0-100 \mu \mathrm{M}$ $\mathrm{PN}$ for $1 \mathrm{~h}$ at $37^{\circ} \mathrm{C}$, and $10 \mathrm{~mm}$ DTT was applied immediately thereafter. Caspase- 9 activity was assessed using the fluorogenic Ac-LEHD-AFC assay (see Materials and Methods). PN strongly inhibited caspase- 9 activity, and this was almost completely reversed by the DTT treatment (Fig. 4C). These data suggest that, at least in a cell-free system, $\mathrm{PN}$ inhibits the activity of both caspase- 3 and caspase- 9 by reversible cysteinyl oxidation.

Next, we sought to determine whether PN can inactivate caspase- 3 in cultured cortical neurons otherwise committed to apoptosis. To this end, we developed and optimized two separate paradigms. In the first, $\mathrm{PN}$ formation was elicited without caspase-3 activation, and in the second, caspase- 3 activation was elicited without PN formation. Combining these should demonstrate whether caspase- 3 activation is affected by PN.

\section{Staurosporine treatment causes caspase-3-dependent cell death without protein nitration}

First, the cultures were treated with $1 \mu \mathrm{M}$ staurosporine, a general protein kinase inhibitor that induces caspase-3-mediated death in murine cortical cell cultures (Koh et al., 1995). The cells were harvested at different times (3-24 h) and assayed in Western blots for cleaved (active) caspase-3 (Fig. 5A) and for 3-nitrotyrosine (Fig. $5 B)$. Staurosporine-induced progressive caspase- 3 cleavage $(\sim 17 \mathrm{kDa}$ band in Fig. $5 A$ ) was observable until $24 \mathrm{~h}$, and this occurred in the absence of 3-nitrotyrosine formation (Fig. 5B). TUNEL staining $24 \mathrm{~h}$ after staurosporine treatment revealed a marked increase in DNA fragmentation (Fig. $5 C$, right panel), and this was concomitant with a progressive reduction in cell viability as assayed
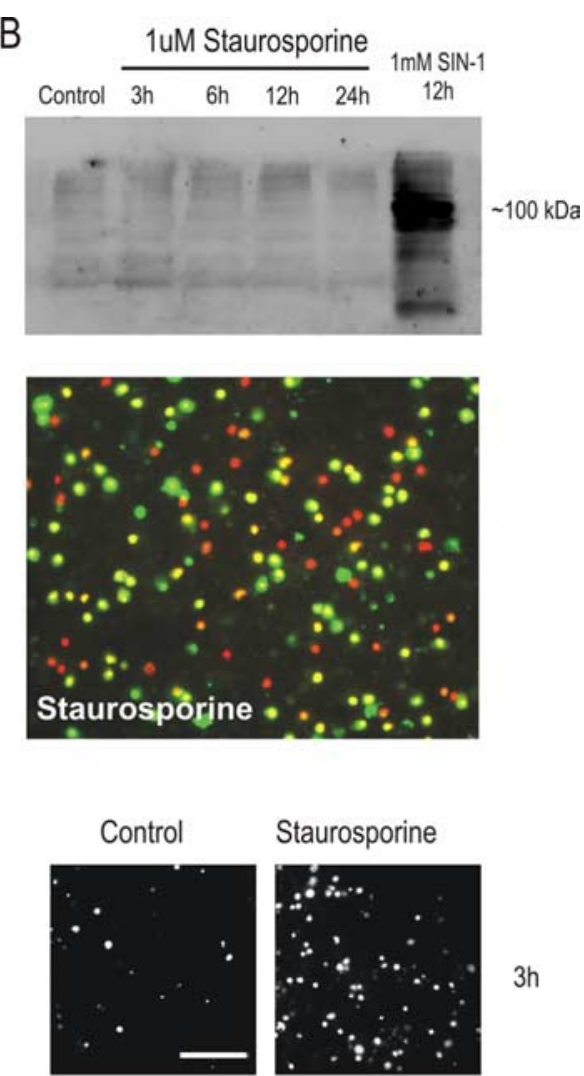

Staurosporine

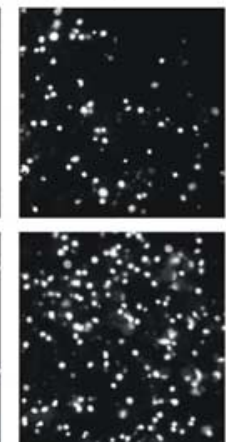

$3 h$

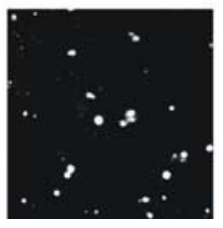

$24 \mathrm{~h}$
$24 h$

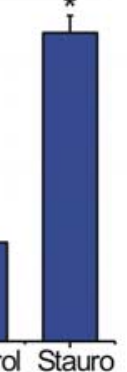

Figure 5. Dependence of staurosporine-mediated neuronal death on caspase-3 activation but not on protein nitration. $\boldsymbol{A}, \boldsymbol{B}$, control in $\boldsymbol{B}$. C, Fluorescent TUNEL labeling (green) of staurosporine-treated cultures (right) compared with controls (left). Cells were counterstained with Hoescht (red) to show all nuclei ( $n=3$ experiments). Scale bar, $100 \mu \mathrm{m}$. $\boldsymbol{D}$, Cell death resulting from staurosporine treatment (Stauro) at the indicated times ( $n=4$ experiments; asterisks, different from control at $p<0.05$ ). Right panels, Representative PI fluorescence images at $24 \mathrm{~h}$ from the indicated conditions. Scale bar, $100 \mu \mathrm{m}$.

with PI at 3 and $24 \mathrm{~h}$ (Fig. 5D). Thus, staurosporine induces caspase- 3 activation and cell death without protein nitration.

\section{SIN-1 treatment causes protein nitration without caspase- 3 activation}

Next, sister cortical cultures were treated with $1 \mathrm{~mm}$ SIN-1. This treatment did not induce caspase- 3 activation (Fig. 6A), whereas it induced robust protein nitration at all time points between 3 and $24 \mathrm{~h}$ (Fig. 6B). This same concentration of SIN-1 did not cause detectable TUNEL staining in the cultures (Fig. 6C, middle panel), indicating that $1 \mathrm{~mm} \mathrm{SIN-1}$ can be used to elicit protein nitration without TUNEL-detectable DNA damage. Notably, DNA fragmentation was not seen until the concentration of SIN-1 was raised to $3 \mathrm{~mm}$ (Fig. $6 \mathrm{C}$, right panel). Concomitant cell viability assays revealed that $1 \mathrm{~mm}$ SIN-1 was not toxic to the cultures, whereas 3-10 mM SIN-1 significantly increased cell death at both 3 and $24 \mathrm{~h}$ after treatment (Fig. 6D). Thus, $1 \mathrm{~mm}$ SIN-1 elicits protein nitration without inducing caspase- 3 activation or cell death. 
A

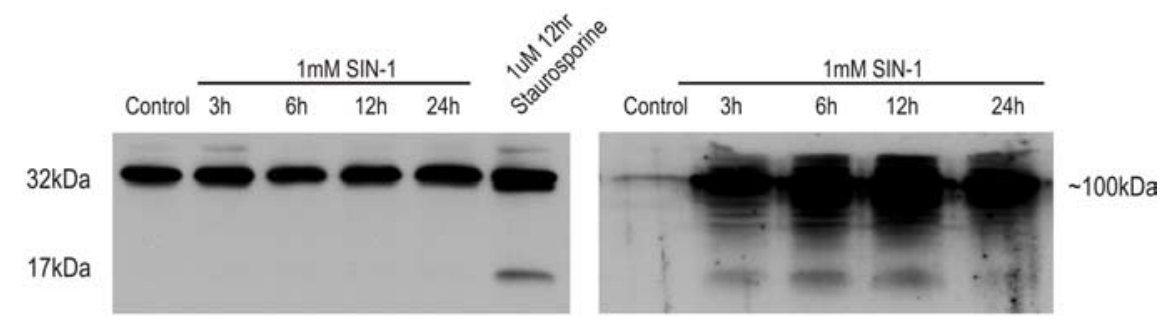

C
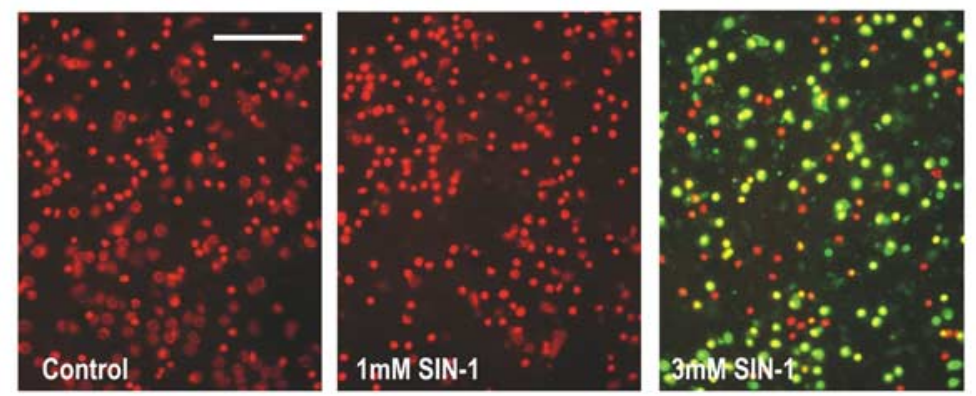

D
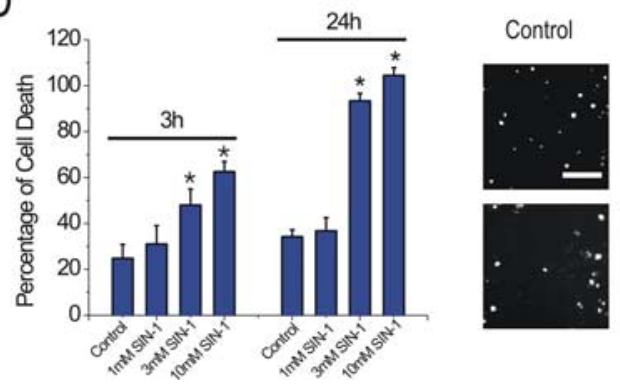

$1 \mathrm{mM}$ SIN-1
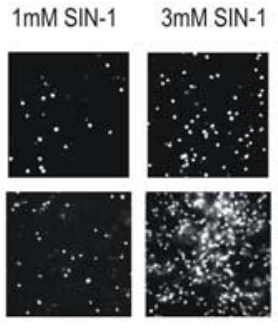

Figure 6. Dependence of SIN-1-mediated neuronal death on protein nitration but without caspase-3 activation. $\boldsymbol{A}, \boldsymbol{B}$, Immunoblots using anti-caspase-3 (pro- and active/cleaved forms) and anti-3-nitrotyrosine antibodies of whole-cell lysates from cultures treated with $1 \mu \mathrm{m}$ staurosporine for the indicated times. Active caspase-3 immunoreactivity ( $\sim 17 \mathrm{kDa}$ band) does not increase at any time $(\boldsymbol{A} ; n=2$ experiments), whereas anti-NT staining increases at all time points assayed ( $\boldsymbol{B} ; n=2$ experiments). Staurosporine (1 $\mu \mathrm{m}$ ) was used as a positive control in $\boldsymbol{A}$. C, DNA fragmentation observed with TUNEL staining (green) after 1 and 3 mM SIN-1 (middle and right panels, respectively). Cells were counterstained with Hoescht (red) to visualize nuclei $(n=3$ experiments). Scale bar, $100 \mu \mathrm{m}$. D. Cell death at 3 and $24 \mathrm{~h}$ resulting from SIN- 1 treatment at the indicated concentrations ( $n=$ 4 experiments; asterisks, different from control at $p<0.05$ ). Right panels, Representative PI fluorescence images at the indicated times and conditions. Scale bar, $100 \mu \mathrm{m}$.

SIN-1 blocks staurosporine-induced caspase-3 activation and its downstream effects but not cell death

In the context of the above, we next combined the SIN-1 and staurosporine treatments. If $\mathrm{PN}$ formation can inhibit caspasemediated apoptosis, then SIN-1 treatment should inhibit staurosporine-induced caspase-3 activation. Using cultures treated with $1 \mu \mathrm{M}$ staurosporine, we coapplied SIN- 1 at $1 \mathrm{~mm}$, the concentration that nitrated proteins in Figure $6 \mathrm{~B}$ without impacting cell survival as shown in Figure $6 D$. Coapplication of SIN-1 to the cultures abolished staurosporine-induced caspase- 3 cleavage as detected in immunoblots using cells harvested for up to $24 \mathrm{~h}$ after the insult (Fig. 7A).

To test whether the suppression of caspase- 3 cleavage seen in immunoblots corresponded to a reduction in caspase activity, we next examined caspase- 3 activity in the fluorogenic activity assay (see Materials and Methods). Cell lysates were obtained from the cortical cultures $24 \mathrm{~h}$ after treatment with either SIN-1 (1 mM), staurosporine $(1 \mu \mathrm{M}), 1 \mathrm{~mm}$ SIN-1 plus $1 \mu \mathrm{M}$ staurosporine, or HEPES control solution. The lysates were incubated with the

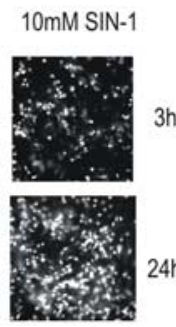

caspase substrate Ac-DEVD-AMC (10 $\mu \mathrm{M})$, and the fluorescence was quantified. Consistent with the results obtained by immunoblotting, only the staurosporine treatment induced a significant increase in caspase- 3 activity (Fig. $7 B)(p<0.05 ; n=$ 4 assays). Caspase- 3 activity was undetectable above control levels in staurosporinetreated cultures that were cotreated with SIN-1. Thus, a PN donor can inhibit caspase activity in otherwise apoptotic primary cell cultures.

To further test for inhibition of caspase-3, we evaluated whether any of its downstream effects were detectable. A direct downstream effect of activated caspase- 3 is the proteolytic cleavage of the $\sim 116 \mathrm{kDa}$ DNA repair enzyme PARP-1 into a smaller $\sim 85 \mathrm{kDa}$ inactive fragment (Lazebnik et al., 1994). Because the response of PARP-1 to DNA damage requires ATP, PARP-1 inhibition by caspases may serve to preserve ATP to enable apoptosis (Stefanis, 2005). If PN inactivates caspase-3, then this should prevent PARP-1 cleavage. To check this, cell lysates obtained from the cortical cultures $12 \mathrm{~h}$ after treatment with $1 \mathrm{~mm}$ SIN-1 in combination with $1 \mu \mathrm{M}$ staurosporine were assayed for PARP-1 cleavage on immunoblots. These cells exhibited the anticipated PARP- 1 band at $\sim 116 \mathrm{kDA}$, but no band corresponding to the cleaved form of PARP-1 ( $~ 85 \mathrm{kDa})$ was observable at any time point assayed (Fig. 7C). As controls for PARP-1 cleavage, we used both cell lysates from staurosporinetreated cortical cultures at $12 \mathrm{~h}$, and from Jurkat T cells treated with camptothecin (PharMingen).

Phosphotidylserine inversion is another hallmark of apoptosis that occurs on the plasma membrane (Fadok et al., 1992). Annexin V is a protein that binds with high affinity to phosphotidylserine and, when conjugated to a visualization agent, can be used to assess apoptotic-like cellular behavior. The application of an annexin V-FITC conjugate to cell cultures treated with $1 \mu \mathrm{M}$ staurosporine resulted in an expected increase in fluorescence (Fig. 7D). The addition of SIN-1 to staurosporine-treated cells significantly attenuated this increase in fluorescence (Fig. 7D), indicating attenuation of phosphatidylserine inversion.

Despite the inhibition of caspase- 3 and its downstream effects, the cotreatment of cells with SIN-1 and staurosporine resulted in a similar extent of TUNEL staining compared with staurosporine treatment alone (Fig. 7E). Quantification of TUNEL staining was then performed by normalizing TUNEL-positive cells to the number of Hoechst-stained cells in each randomly selected field. No significant difference was observed in DNA fragmentation with SIN-1 and staurosporine-cotreated cultures compared with staurosporine treatments alone $(86.5 \pm 5.1 \%$ compared with $82.1 \pm 1.9 \%$ TUNEL-positive cells; from an average of at least 12 random fields per condition). Thus, we conclude that cell death 
A
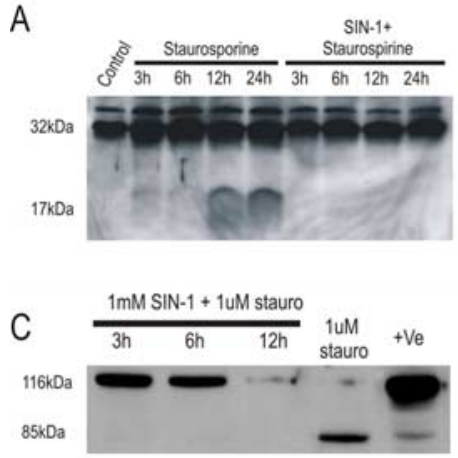

D
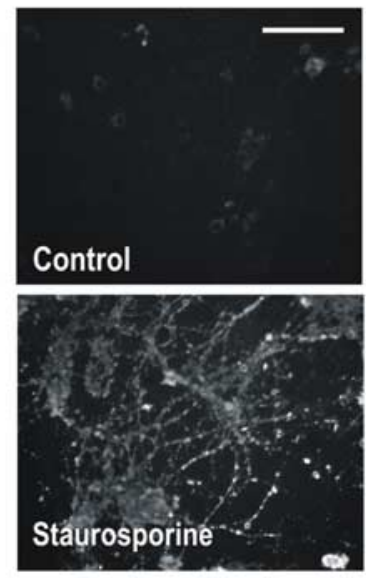

$E$

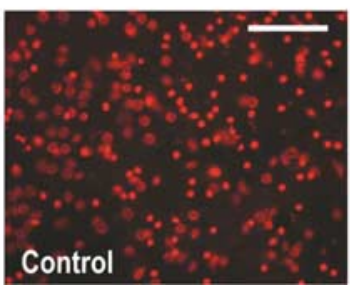

B
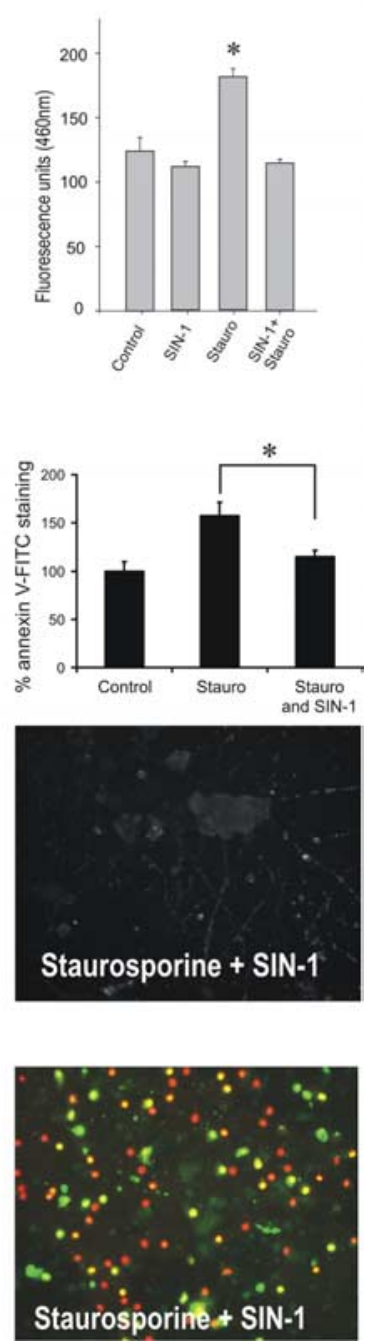

Figure 7. SIN-1 attenuates staurosporine-induced caspase-3 activation and apoptotic events, but not cell death. $\boldsymbol{A}$, Caspase- 3 immunoblot of whole-cell lysates taken at the indicated times after treatment with $1 \mu \mathrm{m}$ staurosporine alone or in combination with $1 \mathrm{~mm}$ SIN-1. Active caspase-3 immunoreactivity ( $\sim 17 \mathrm{kDa}$ band) induced by staurosporine (lanes $2-5$ ) was abolished after the application of $1 \mathrm{~mm}$ SIN-1 (lanes 6-9). B, Caspase-3 activity measurements with Ac-DEVD-AMC using cell lysates taken ( $24 \mathrm{~h}$ ) after the indicated insults ( $n=4$ experiments; asterisk, different from control at $p<0.05)$. C, Effect of staurosporine alone and in combination with SIN-1 on PARP-1 cleavage ( $n=2$ experiments; +Ve indicates positive control using camptothecin-treated Jurkat cells). D, Staining of the cultures using FITC-conjugated annexin V, a label of inverted phosphatidylserine, after treatment with staurosporine alone or in combination with SIN- 1 ( $n=3$ experiments). Scale bar, $50 \mu \mathrm{m}$. For quantification, FITC fluorescence was averaged from several images taken using identical settings, normalized to baseline controls, and expressed as a percentage increase over baseline controls. The application of $1 \mathrm{~mm}$ SIN-1 significantly reduced FITC staining compared with staurosporine treatment alone ( $p<$ $0.05 ; n=4$ experiments per condition). $\boldsymbol{E}$, TUNEL (green) and Hoechst (red) nuclear labeling of cultures after treatment with staurosporine alone or the staurosporine/SIN-1 combination ( $n=3$ experiments per condition). Scale bar, $100 \mu \mathrm{m}$. No significant difference (see Results) is observed in cell death after the application of $1 \mathrm{~mm} \mathrm{SIN-1}$ compared with staurosporine alone (also compare images with Fig. 5C).

in the apoptotic paradigm induced by staurosporine could be shifted by PN to a caspase-independent death.

Lateral FPI causes an increase in peroxynitrite production Our in vitro and ex vivo experiments have shown that $\mathrm{PN}$ is formed in our stretch injury model and, while creating DNA

damage and causing neurotoxicity, it also nitrates, oxidizes, and inactivates key components of caspase-mediated apoptotic mechanisms. To determine the in vivo relevance of these findings, we next set out to examine them further in an animal model of TBI. We selected the lateral FPI model in the rat, an established and validated animal model of TBI (Dixon et al., 1987; McIntosh et al., 1989). Sprague Dawley rats were subjected to a 2.4 atm FPI (see Materials and Methods), an injury severity that, in pilot studies using our apparatus, was the highest impact injury that caused minimal $(\sim 6 \%)$ early (postimpact) mortalities in test animals. Animals that underwent FPI experienced $<30$ s of apnea.

First, we determined whether FPI resulted in peroxynitrite production as had the stretch injury in the cultured neurons. Coronal histological sections were taken from paraffinembedded brains removed from animals $24 \mathrm{~h}$ after FPI. The injured area could be readily visualized in hematoxylin and eosin $(\mathrm{H}+\mathrm{E})$-stained sections when these were viewed at low-power magnification with bright-field optics (Fig. $8 \mathrm{~A}$ ) or with higherpower magnification using phase contrast optics (Fig. 8 B). Coronal sections were also immunostained with anti-3-nitrotyrosine antibodies and visualized with DAB (Fig. 8C). FPI caused increased 3-nitrotyrosine immunoreactivity in a cortical region corresponding to the injured areas as observed using $\mathrm{H}+\mathrm{E}$ staining (Fig. $8 \mathrm{~A}$, black arrows). Animals undergoing sham surgery did exhibit increased 3-nitrotyrosine immunoreactivity in the cortex (data not shown).

To corroborate our histological findings, 2-mm-thick coronal sections were obtained from injured and uninjured animals. Coronal sections were then stained with the mitochondrial dye TTC. This dye is most commonly used in stroke research to delineate infarct size by staining living tissue red (Bederson et al., 1986). Here, it was used to delineate the damaged cortical area in unfixed tissue (Fig. $8 D$, left brain), thus enabling the harvesting of cortical tissue samples from the injury area. Samples from both hemispheres were taken from injured and uninjured shams and run on SDS-PAGE. Western blots using an anti-3-nitrotyrosine antibody with these samples revealed increased levels of nitrotyrosine in the ipsilateral, injured cortex at $32 \mathrm{kDa}$ (Fig. 8 E). However, immunoblots for caspase-3 (both pro- and active/cleaved forms) from the same lysates failed to reveal any increased caspase- 3 activation in the injured cortex (Fig. $8 F$ ). These results suggest that cortical histological damage is associated with mitochondrial dysfunction, PN formation, and lack of caspase-3 activation at $24 \mathrm{~h}$ after FPI.

\section{Peroxynitrite formation after fluid percussion injury is associated with DNA damage and neurodegeneration}

To determine the cellular localization of peroxynitrite formation in vivo, we next doubly immunostained $25 \mu \mathrm{m}$ coronal sections taken from animals subjected to FPI using antibodies against 3-nitrotyrosine and anti-NeuN (Mullen et al., 1992). There was marked colocalization of both markers, indicating protein nitration by PN in neurons (Fig. 9A, top panels). Notably, anti-3nitrotyrosine staining was not observed in the contralateral (uninjured) cortex (Fig. 9A, bottom panels). Higher-power confocal images clearly showed that the nitration was preferentially distributed in cytoplasm of injured neurons in the ipsilateral cortex (Fig. 9A). 3-Nitrotyrosine-positive neurons also demonstrated irregular nuclear morphology when compared with neurons in the contralateral cortex, suggestive of ongoing degenerative processes (data not shown).

We next examined whether neurons that exhibit peroxynitrite formation after in vivo TBI also exhibit DNA damage as detect- 
A

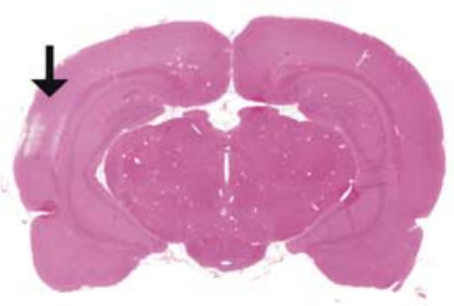

C

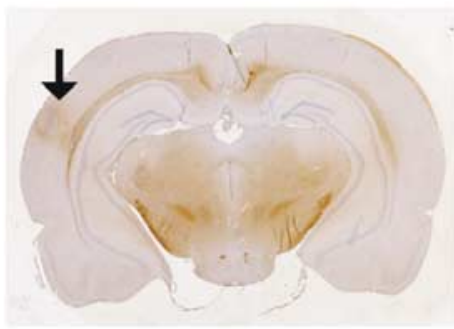

$E$

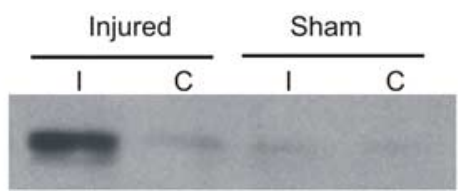

B

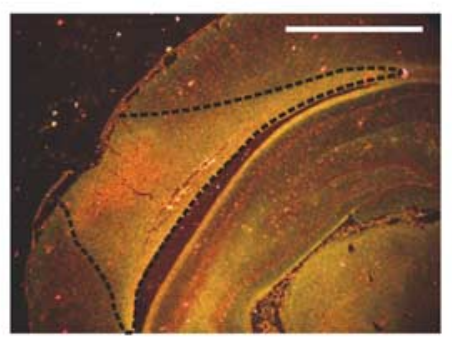

D

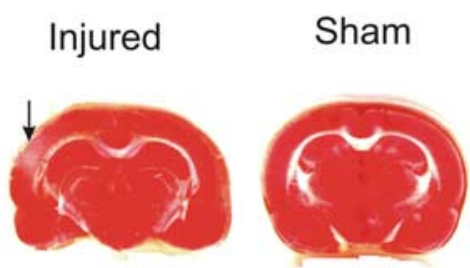

$\mathrm{F}$

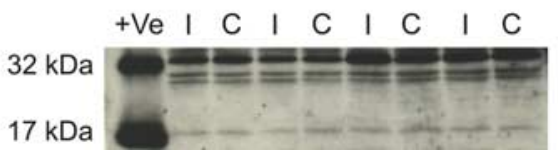

Figure 8. Lateral fluid percussion TBI induces protein nitration in the rat brain. $\boldsymbol{A}, \mathrm{H}+\mathrm{E}$-stained coronal section demonstrating gross histological damage (arrow) $7 \mathrm{~d}$ after fluid percussion injury. $\boldsymbol{B}$, Low-power view (phase contrast) of the area of cortical injury $(24 \mathrm{~h})$ after lateral fluid percussion injury in the rat. The dotted line outlines the injury area. Scale bar, $500 \mu \mathrm{m}$. C, Anti-NT staining of same section, indicating increased NT staining (arrow) in the injured area. Immunoreactivity was visualized using DAB, and sections were counterstained using cresyl violet. $\boldsymbol{A}-\boldsymbol{C}$ are representative of at least six animals per experiment. $\boldsymbol{D}$, Representative brain sections from sham and injured animals stained with TTC to delineate the injury site (arrow) for protein harvest. $\boldsymbol{E}$, Immunoblots using anti-3-nitrotyrosine antibodies from injured (I) and contralateral (C) cortex taken $24 \mathrm{~h}$ after TBI or control (sham) animals ( $n=6$ animals/experiment). Notably, this monoclonal antibody ( $4 \mu \mathrm{g} / \mathrm{ml}$; BioMol) detects this $\sim 30 \mathrm{kDa}$ band more selectively than the polyclonal antibody (Upstate Biotechnology) used in the tissue culture experiments (Fig. 2D). $\boldsymbol{F}$, Immunoblot of caspase-3 (both pro- and active/cleaved forms) from brain lysates taken at $24 \mathrm{~h}$ from cortex ipsilateral (I) and contralateral (C) to FPI in four animals subjected to a 2.4 atm injury. Positive control, $20 \mu \mathrm{g}$ recombinant human caspase-3 (Sigma).

able by TUNEL staining. Paraffin-embedded 7 - $\mu \mathrm{m}$-thick coronal sections were doubly immunostained with TUNEL and with anti-3-nitrotyrosine. TUNEL staining indicative of DNA fragmentation was colocalized with anti-3nitrotyrosine-stained neurons (Fig. 9B, top panels). However, no detectable TUNEL or anti-3-nitrotyrosine staining was observed in the contralateral cortex (Fig. 9B, bottom panels). Together, these results demonstrate a neuronal pathology in rats subjected to FPI that is similar to that observed in the stretch plus $30 \mu \mathrm{M}$ NMDA cell culture model of TBI in which peroxynitrite formation is associated with DNA degradation and neurodegeneration.

\section{Cysteinyl oxidation inhibits caspase- 3 activity after fluid percussion injury}

Having shown that $\mathrm{PN}$ can inactivate caspases in an ex vivo system (Fig. 4) and in cultured neurons (Figs. 5-7), we next determined whether the PN formed in TBI has similar effects in vivo.

To determine the levels of caspase- 3 activity after FPI, injured and uninjured cortical regions were harvested $24 \mathrm{~h}$ after injury. Forty micrograms of each sample were then incubated with either recombinant caspase-9 (Sigma), DTT, the caspase inhibitor $\mathrm{z}$-VAD-fmk (EMD Biosciences), or DMSO as a control for $1 \mathrm{~h}$ at $37^{\circ} \mathrm{C}$. After incubation, $40 \mu \mathrm{M}$ Ac-DEVD$\mathrm{AMC}$ was added, and the fluorescence was observed. It is expected that the application of $\mathrm{z}$-VAD-fmk should produce activity measurements commensurate with minimum caspase-3 activity levels, whereas caspase- 9 treatment should verify that caspase-3 pathway was able to be activated.

Analysis of samples taken from contralateral (uninjured) cortex of animals after TBI revealed that DMSO-treated samples elicited Ac-DEVD-AMC fluorescence values similar to those of samples treated with $\mathrm{z}$-VAD-fmk, suggesting that baseline active caspase- 3 level in uninjured cortex is low (Fig. 10A, left panel). The addition of caspase- 9 to samples from uninjured cortex increased Ac-DEVD-AMC fluorescence over the ensuing hours, indicating that procaspase- 3 was present and available to be activated. The addition of DTT had relatively little effect on caspase- 3 activity in the uninjured cortex compared with the ipsilateral cortex, suggesting that in uninjured cortex, caspase- 3 is in a relatively nonoxidized state (Fig. 10 A, left panel).

When samples taken from the ipsilateral (injured) cortex were processed and analyzed identically to the uninjured samples, similar results were obtained for the baseline (DMSO control) and z-VAD-fmk treatment, indicating that in the absence of exogenous, recombinant caspase- 9 , no significant caspase- 3 activity was observable from injured cortex (Fig. 10 A, right panel). Also, treatment with exogenous caspase-9 increased Ac-DEVD-AMC fluorescence to levels similar to those measured in the contralateral cortex, suggesting that procaspase- 3 is also available for activation in the injured cortex. However, unlike in contralateral cortex, the addition of DTT increased the Ac-DEVD-AMC fluorescence significantly (Fig. $10 \mathrm{~A}$, right panel), demonstrating that a substantial amount of caspase- 3 must have existed in an inhibited, oxidized state after FPI. As shown in the ex vivo studies (Fig. $4 B$ ), restoration of function using DTT suggests that an oxidative modification of cysteine residues is responsible for the inhibition of caspases after FPI.

Our ex vivo experiments suggested that caspase- 9 activity was also affected by PN (Fig. 7C). To examine whether the same occurs in FPI, we examined its effects on endogenous caspase-9 activity. Samples taken from uninjured (contralateral) and injured (ipsilateral) brain cortices were incubated with DTT or with z-VAD-fmk. Unlike with caspase-3, no significant differences in endogenous caspase- 9 activity were observed between injured and uninjured cortex with or without DTT. This suggests that, in vivo, $\mathrm{PN}$ affects caspase-3-like activity preferentially over caspase-9-like activity. This in vivo result is supported by our finding that PN preferentially nitrated procaspase-3 but not caspase-9 in the in vitro stretch model (Fig. $3 A, C$ ). It is possible that native caspases might have different susceptibilities to inactivation by $\mathrm{PN}$. 


\section{Discussion}

Our data, derived from exvivo assays, in vitro neuronal stretch, and in vivo FPI, demonstrate inactivation of classical apoptosis mediated by PN mostly by targeting caspase- 3 in TBI. Furthermore, this inactivation of apoptosis was shown to be primarily dependent on cysteinyl oxidation and inhibition of proteolytic function. Thus, $\mathrm{PN}$ acts to switch off caspase-mediated effects and apoptotic mechanisms in TBI.

Our data do not exclude a role for apoptosis in TBI, nor do they exclude a role for other mechanisms of neuronal damage. Indeed, many neurotoxic signaling mechanisms have been implicated after TBI. Caspases (Yakovlev et al., 1997; Clark et al., 1999, 2000; Beer et al., 2000, 2001; Knoblach et al., 2002), calpains (Pike et al., 1998, 2000; Buki et al., 1999; Knoblach et al., 2004), PARP-1 (LaPlaca et al., 2001; Besson et al., 2003; Hortobagyi et al., 2003), reactive nitrogen species (Mesenge et al., 1998; Hall et al., 2004), and reactive oxygen species (Marklund et al., 2001a,b) have all been suggested to play key roles in TBI. In addition, it is recognized that cross talk may exist between signaling pathways. For example, calpains can proteolytically inactivate procaspase- 9 in neurons after NO application (Volbracht et al., 2005), NO can inhibit caspase-3 directly in human umbilical vein endothelial cells (Dimmeler et al., 1997), and caspase-3 can proteolytically inactivate PARP-1 (Lazebnik et al., 1994). Our present study contributes to unraveling this complexity by elucidating the interplay between caspasemediated apoptosis and PN-mediated damage. The results here suggest that treating apoptosis alone may be insufficient, because PN-mediated damage would be unaffected. Similarly, treating PN-mediated effects using ROS scavengers or NO inhibitors may be insufficient, because as PN levels drop, proteolytic apoptotic mechanisms could be disinhibited and come into play. Thus, at a minimum, multimodality therapy in TBI should focus on anti-oxidant and anti-apoptotic strategies.

Other studies have indirectly supported the possibility that apoptosis may be oxidatively inhibited after TBI. For example, a study by Lewen et al. (2001) demonstrated that scavenging reactive oxygen species using $\alpha$-phenyl-tert- $N$-butyl nitrone actually increased levels of detectable DNA fragmentation after TBI at $24 \mathrm{~h}$. In this scenario, it is possible that the inhibition of peroxynitrite formation caused a disinhibition of caspase- 3 and permitted apoptotic DNA degradation to occur. Moreover, other studies have noted cytochrome $c$ release into the cytoplasm, and yet no protection with the caspase inhibitor z-DEVD-fmk (Sullivan et al., 2002). Together with our results, these studies support a therapeutic approach in which coinhibition of both oxidative and proteolytic mechanisms are necessary. tion in the overlay panels.

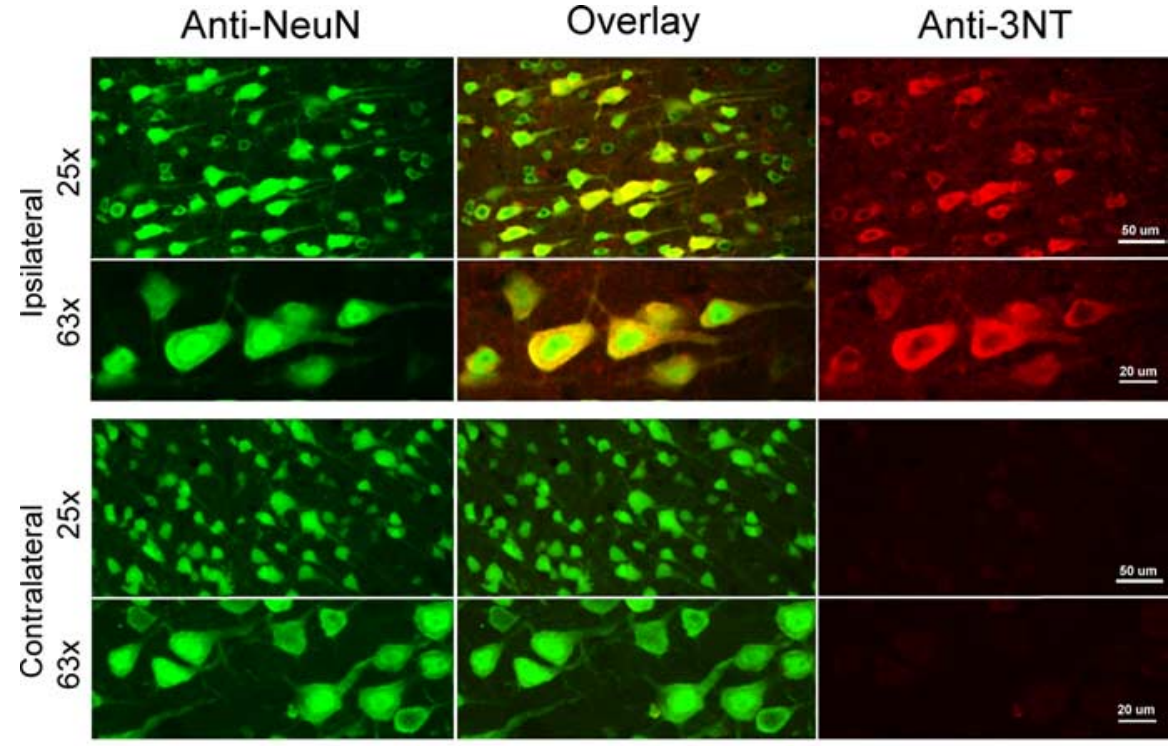

TUNEL

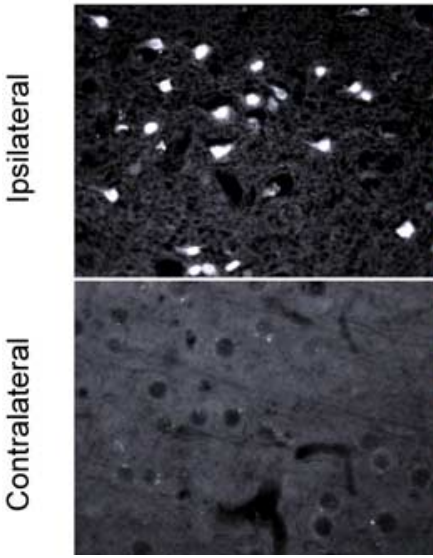

Anti-3-NT
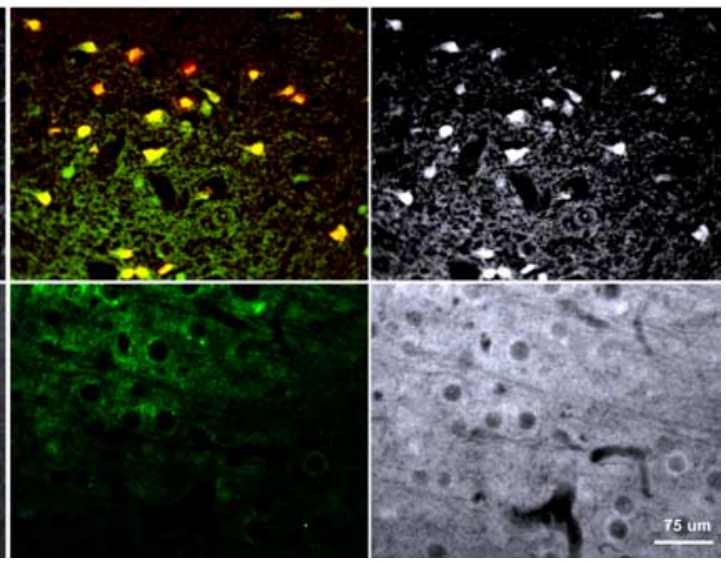

Figure 9. Lateral fluid percussion TBI induces TUNEL and protein nitration in neurons in vivo. Rats underwent a 2.4 atm FPI. The brains were extracted at $24 \mathrm{~h}$ and sectioned. $\boldsymbol{A}$, Confocal imaging of the colocalization of Anti-3-NT staining with the neuronal (contralateral, bottom panels) ( $n=3$ experiments). At higher power $(63 \times)$, the anti-3-nitrotyrosine staining is

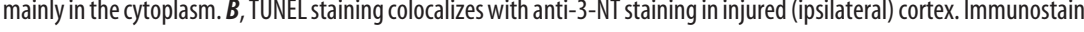

In vivo, it is possible that different injury mechanisms may dominate in different cell populations or brain regions. For example, Figure $8 C$ illustrates that anti-nitrotyrosine staining, a surrogate measure of PN activity, is not evenly distributed in all brain regions or among all neurons in the injured areas (Fig. $9 A, B)$. Thus, it is possible that, because of this heterogeneous injury pattern, PN-mediated damage may occur in some cells, whereas apoptotic mechanisms could predominate in others (Conti et al., 1998). Supporting evidence is provided by Pike et al. (1998), who demonstrated that, while evidence of caspase-3 proteolysis is absent in the cortex after TBI, significant increases in caspase-3 proteolysis was observed in the hippocampus. Moreover, our data do not rule out the possibility that, whereas PN formation occurs acutely, additional apoptotic triggers could arise in a delayed manner and initiate delayed apoptosis beyond the times studied here. Such explanations could account for previous studies in which caspase-3 activity was reported after TBI in 
A

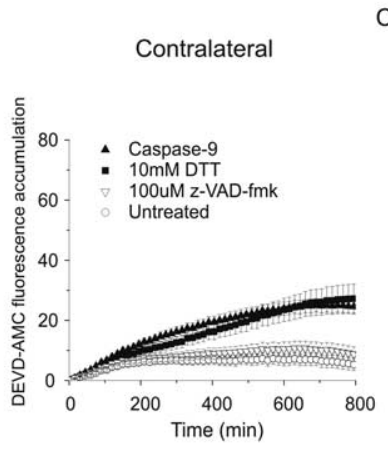

Caspase-3

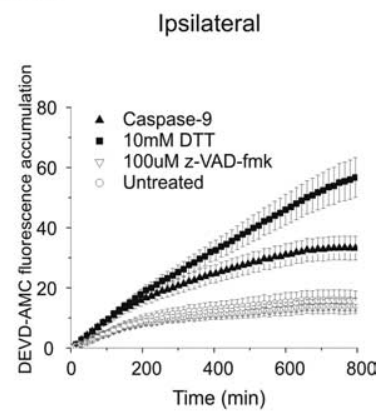

B

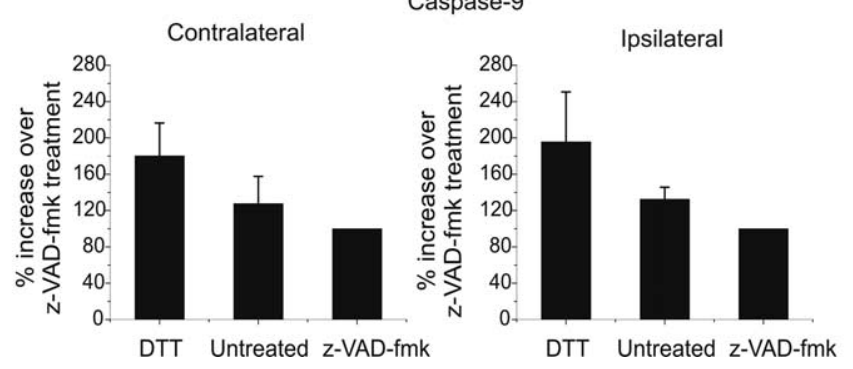

Figure 10. Caspase inhibition via cysteinyl oxidation in FPI affects caspase-3 preferentially to caspase-9. Cortical lysates were harvested at $24 \mathrm{~h}$ from FPI injured animals (2.4 atm) and assayed for caspase-3 and caspase- 9 activity using Ac-DEVD-AMC and AC-LEHD-AFC, respectively ( $n=6$ animals). $A$, Effects of incubation with DTT, activated caspase- 9 ( $3 \mathrm{U})$, or z-VADfmk on caspase-3 activity in cortex ipsilateral (right) and contralateral (left) to the FPI. DTTtreated samples exhibited significantly higher levels of caspase-3 proteolytic activity in the ipsilateral cortex compared with the contralateral cortex. $\boldsymbol{B}$, Effects of incubation with DTT or z-VAD-fmk on caspase-9 activity in cortex ipsilateral (right) and contralateral (left) to the FPI. No significant differences in caspase- 9 activity between the ipsilateral and contralateral cortex were observed with any treatment assayed.

animal studies (Yakovlev et al., 1997; Beer et al., 2000; Clark et al., 2000; Knoblach et al., 2002) and human studies (Clark et al., 1999). However, others suggest that procaspase- 3 cleavage is not detectable even at $24 \mathrm{~h}$ after FPI (Knoblach et al., 2004). Notably, Knoblach et al. (2002) and Clark et al. (2000) noted that many TUNEL-stained neurons in TBI were not colocalized with active caspase-3, supporting the notion that caspase-3 activity, if it occurs, might arise from only a subset of a larger population of neurons in which DNA degradation occurs. In the end, all of these alternatives further support the prediction that a strategy using a single treatment will be less effective than a treatment strategy that targets both PN and caspase-dependent mechanisms. Although targeting multiple mechanisms is an intuitive approach to improving the success of TBI therapies, our work additionally provides specifics as to which mechanisms should be targeted.

Our findings show that $\mathrm{PN}$ formation in neurons subjected to TBI can act as a molecular switch that predisposes neurons to die via oxidative mechanisms by simultaneously inhibiting proteolytic cell death pathways. An implication of these findings is that, if caspases are being inhibited, then other proteolytic pathways of relevance to TBI may likewise be affected. Of particular interest are calpains which, like caspases, are cysteine proteases and have been implicated mediating secondary damage in TBI. Accordingly, an oxidative inhibition of calpains in hippocampi in a model of Alzheimer's disease is reported (Marcum et al., 2005), although the extent of oxidation-mediated inhibition of calpains in TBI remains to be seen.
Although our work supports a role for PN as a key oxidant molecule in TBI, we cannot exclude contributions from other oxidative mechanisms in caspase inhibition. NO-mediated inhibition of caspases is a possibility. Initially reported by Mannick et al. (1994) in B lymphocytes, J. Li et al. (1997) later demonstrated in an ex vivo system a pan-inhibitory effect of $\mathrm{NO}$ on caspases -1, $-2,-3,-4,-6,-7$, and -8 . However, PN inhibits caspases more potently compared with NO (Mohr et al., 1997), and NO produced after TBI may preferentially interact with superoxide and form PN before peptide interaction (Cherian and Robertson, 2003).

The origin of PN in TBI also remains to be determined. In the in vitro stretch model, it is likely produced in the neurons as $\mathrm{PN}$ formation is triggered by the NMDA application (Arundine et al., 2004). However, in whole animal models and in human cases of TBI, it has also been demonstrated that inducible nitric oxide synthase (iNOS) production of NO increases after injury (Wada et al., 1998). Recently, inhibition of neuronal NOS and endothelial NOS has been demonstrated to reduce nitrotyrosine staining (Gahm et al., 2005), although it remains to be determined whether inhibition of iNOS will yield similar results. It may be that the location of superoxide radical is the key to the localization of PN formation, because NO is more freely diffusible through plasma membranes. Superoxide forms in stretched neurons (Arundine et al., 2004) but in vivo may arise in other cell types as well.

In conclusion, our finding that $\mathrm{PN}$ acts to switch off caspasemediated apoptosis reveals that there is cross talk among key neurotoxic signaling mechanisms in TBI. Unraveling these signaling pathways may be essential to establishing future rational therapeutic approaches. In TBI, both anti-oxidant and antiapoptotic strategies may be the minimum necessary to reduce traumatic brain damage. Our findings likely have implications to other acute CNS disorders in which multiple pathways have been implicated as being causative in mediating neuronal damage.

\section{References}

Amar AP, Levy ML (1999) Pathogenesis and pharmacological strategies for mitigating secondary damage in acute spinal cord injury. Neurosurgery 44:1027-1039.

Arundine M, Chopra GK, Wrong A, Lei S, Aarts MM, MacDonald JF, Tymianski M (2003) Enhanced vulnerability to NMDA toxicity in sublethal traumatic neuronal injury in vitro. J Neurotrauma 20:1377-1395.

Arundine M, Aarts M, Lau A, Tymianski M (2004) Vulnerability of central neurons to secondary insults after in vitro mechanical stretch. J Neurosci 24:8106-8123.

Bao F, Liu D (2003) Peroxynitrite generated in the rat spinal cord induces apoptotic cell death and activates caspase-3. Neuroscience 116:59-70.

Beattie MS, Farooqui AA, Bresnahan JC (2000) Rev of current evidence for apoptosis after spinal cord injury. J Neurotrauma 17:915-925.

Beckman JS, Koppenol WH (1996) Nitric oxide, superoxide, and peroxynitrite: the good, the bad, and ugly. Am J Physiol 271:C1424-C1437.

Bederson JB, Pitts LH, Germano SM, Nishimura MC, Davis RL, Bartowski HM (1986) Evaluation of 2,3,5-triphenyltetrazolium chloride as a stain for detection and quantification of experimental cerebral infarction in rats. Stroke 17:1304-1308.

Beer R, Franz G, Srinivasan A, Hayes RL, Pike BR, Newcomb JK, Zhao X, Schmutzhard E, Poewe W, Kampfl A (2000) Temporal profile and cell subtype distribution of activated caspase- 3 following experimental traumatic brain injury. J Neurochem 75:1264-1273.

Beer R, Franz G, Krajewski S, Pike BR, Hayes RL, Reed JC, Wang KK, Klimmer C, Schmutzhard E, Poewe W, Kampfl A (2001) Temporal and spatial profile of caspase 8 expression and proteolysis after experimental traumatic brain injury. J Neurochem 78:862-873.

Besson VC, Croci N, Boulu RG, Plotkine M, Marchand-Verrecchia C (2003) Deleterious poly(ADP-ribose)polymerase-1 pathway activation in traumatic brain injury in rat. Brain Res 989:58-66. 
Bolanos JP, Almeida A, Stewart V, Peuchen S, Land JM, Clark JB, Heales SJ (1997) Nitric oxide-mediated mitochondrial damage in the brain: mechanisms and implications for neurodegenerative diseases. J Neurochem 68:2227-2240.

Bruno VMG, Goldberg MP, Dugan LL, Giffard RG, Choi DW (1994) Neuroprotective effect of hypothermia in cortical cultures exposed to oxygenglucose deprivation or excitatory amino acids. J Neurochem 63:1398-1406.

Buki A, Siman R, Trojanowski JQ, Povlishock JT (1999) The role of calpainmediated spectrin proteolysis in traumatically induced axonal injury. J Neuropathol Exp Neurol 58:365-375.

Buki A, Okonkwo DO, Wang KK, Povlishock JT (2000) Cytochrome $c$ release and caspase activation in traumatic axonal injury. J Neurosci 20:2825-2834.

Cherian L, Robertson CS (2003) L-arginine and free radical scavengers increase cerebral blood flow and brain tissue nitric oxide concentrations after controlled cortical impact injury in rats. J Neurotrauma 20:77-85.

Clark RS, Kochanek PM, Chen M, Watkins SC, Marion DW, Chen J, Hamilton RL, Loeffert JE, Graham SH (1999) Increases in Bcl-2 and cleavage of caspase- 1 and caspase- 3 in human brain after head injury. FASEB J 13:813-821.

Clark RS, Kochanek PM, Watkins SC, Chen M, Dixon CE, Seidberg NA, Melick J, Loeffert JE, Nathaniel PD, Jin KL, Graham SH (2000) Caspase- 3 mediated neuronal death after traumatic brain injury in rats. J Neurochem 74:740-753.

Conti AC, Raghupathi R, Trojanowski JQ, McIntosh TK (1998) Experimental brain injury induces regionally distinct apoptosis during the acute and delayed posttraumatic period. J Neurosci 18:5663-5672.

David JC, Yamada KA, Bagwe MR, Goldberg MP (1996) AMPA receptor activation is rapidly toxic to cortical astrocytes when desensitization is blocked. J Neurosci 16:200-209.

Dawson VL, Dawson TM (2004) Deadly conversations: nuclearmitochondrial cross-talk. J Bioenerg Biomembr 36:287-294.

Dimmeler S, Haendeler J, Nehls M, Zeiher AM (1997) Suppression of apoptosis by nitric oxide via inhibition of interleukin-1beta-converting enzyme (ICE)-like and cysteine protease protein (CPP)-32-like proteases. J Exp Med 185:601-607.

Dixon CE, Lyeth BG, Povlishock JT, Findling RL, Hamm RJ, Marmarou A, Young HF, Hayes RL (1987) A fluid percussion model of experimental brain injury in the rat. J Neurosurg 67:110-119.

Estevez AG, Radi R, Barbeito L, Shin JT, Thompson JA, Beckman JS (1995) Peroxynitrite-induced cytotoxicity in PC12 cells: evidence for an apoptotic mechanism differentially modulated by neurotrophic factors. J Neurochem 65:1543-1550.

Estevez AG, Spear N, Manuel SM, Radi R, Henderson CE, Barbeito L, Beckman JS (1998) Nitric oxide and superoxide contribute to motor neuron apoptosis induced by trophic factor deprivation. J Neurosci 18:923-931.

Faden AI (2002) Neuroprotection and traumatic brain injury: theoretical option or realistic proposition. Curr Opin Neurol 15:707-712.

Fadok VA, Voelker DR, Campbell PA, Cohen JJ, Bratton DL, Henson PM (1992) Exposure of phosphatidylserine on the surface of apoptotic lymphocytes triggers specific recognition and removal by macrophages. J Immunol 148:2207-2216.

Fiskum G (2000) Mitochondrial participation in ischemic and traumatic neural cell death. J Neurotrauma 17:843-855.

Gahm C, Danilov A, Holmin S, Wiklund PN, Brundin L, Mathiesen T (2005) Reduced neuronal injury after treatment with NG-nitro-L-arginine methyl ester (L-NAME) or 2-sulfo-phenyl-N-tert-butyl nitrone (S-PBN) following experimental brain contusion. Neurosurgery 57:1272-1281.

Green DR, Reed JC (1998) Mitochondria and apoptosis. Science 281:1309-1312.

Hall ED, Detloff MR, Johnson K, Kupina NC (2004) Peroxynitritemediated protein nitration and lipid peroxidation in a mouse model of traumatic brain injury. J Neurotrauma 21:9-20.

Hill IE, Murray C, Richard J, Rasquinha I, MacManus JP (2000) Despite the internucleosomal cleavage of DNA, reactive oxygen species do not produce other markers of apoptosis in cultured neurons. Exp Neurol 162:73-88.

Hortobagyi T, Gorlach C, Benyo Z, Lacza Z, Hortobagyi S, Wahl M, Harkany $\mathrm{T}$ (2003) Inhibition of neuronal nitric oxide synthase-mediated activation of poly(ADP-ribose) polymerase in traumatic brain injury: neuroprotection by 3-aminobenzamide. Neuroscience 121:983-990.
Ikonomidou C, Turski L (2002) Why did NMDA receptor antagonists fail clinical trials for stroke and traumatic brain injury? Lancet Neurol 383-386

Ischiropoulos H, Zhu L, Chen J, Tsai M, Martin JC, Smith CD, Beckman JS (1992) Peroxynitrite-mediated tyrosine nitration catalyzed by superoxide dismutase. Arch Biochem Biophys 298:431-437.

Joshi CN, Jain SK, Murthy PS (2004) An optimized triphenyltetrazolium chloride method for identification of cerebral infarcts. Brain Res Brain Res Protoc 13:11-17.

Kluck RM, Bossy-Wetzel E, Green DR, Newmeyer DD (1997) The release of cytochrome $\mathrm{c}$ from mitochondria: a primary site for $\mathrm{Bcl}-2$ regulation of apoptosis. Science 275:1132-1136.

Knoblach SM, Nikolaeva M, Huang X, Fan L, Krajewski S, Reed JC, Faden AI (2002) Multiple caspases are activated after traumatic brain injury: evidence for involvement in functional outcome. J Neurotrauma 19:1155-1170.

Knoblach SM, Alroy DA, Nikolaeva M, Cernak I, Stoica BA, Faden AI (2004) Caspase inhibitor z-DEVD-fmk attenuates calpain and necrotic cell death in vitro and after traumatic brain injury. J Cereb Blood Flow Metab 24:1119-1132.

Koh JY, Wie MB, Gwag BJ, Sensi SL, Canzoniero LM, Demaro J, Csernansky C, Choi DW (1995) Staurosporine-induced neuronal apoptosis. Exp Neurol 135:153-159.

LaPlaca MC, Zhang J, Raghupathi R, Li JH, Smith F, Bareyre FM, Snyder SH, Graham DI, McIntosh TK (2001) Pharmacologic inhibition of poly(ADP-ribose) polymerase is neuroprotective following traumatic brain injury in rats. J Neurotrauma 18:369-376.

Lazebnik YA, Kaufmann SH, Desnoyers S, Poirier GG, Earnshaw WC (1994) Cleavage of poly(ADP-ribose) polymerase by a proteinase with properties like ICE. Nature 371:346-347.

Lewen A, Skoglosa Y, Clausen F, Marklund N, Chan PH, Lindholm D, Hillered L (2001) Paradoxical increase in neuronal DNA fragmentation after neuroprotective free radical scavenger treatment in experimental traumatic brain injury. J Cereb Blood Flow Metab 21:344-350.

Li J, Billiar TR, Talanian RV, Kim YM (1997) Nitric oxide reversibly inhibits seven members of the caspase family via S-nitrosylation. Biochem Biophys Res Commun 240:419-424.

Li P, Nijhawan D, Budihardjo I, Srinivasula SM, Ahmad M, Alnemri ES, Wang X (1997) Cytochrome c and dATP-dependent formation of Apaf$1 /$ caspase- 9 complex initiates an apoptotic protease cascade. Cell 91:479-489.

Mannick JB, Asano K, Izumi K, Kieff E, Stamler JS (1994) Nitric oxide produced by human $\mathrm{B}$ lymphocytes inhibits apoptosis and Epstein-Barr virus reactivation. Cell 79:1137-1146.

Marcum JL, Mathenia JK, Chan R, Guttmann RP (2005) Oxidation of thiolproteases in the hippocampus of Alzheimer's disease. Biochem Biophys Res Commun 334:342-348.

Marklund N, Clausen F, McIntosh TK, Hillered L (2001a) Free radical scavenger posttreatment improves functional and morphological outcome after fluid percussion injury in the rat. J Neurotrauma 18:821-832.

Marklund N, Clausen F, Lewander T, Hillered L (2001b) Monitoring of reactive oxygen species production after traumatic brain injury in rats with microdialysis and the 4-hydroxybenzoic acid trapping method. J Neurotrauma 18:1217-1227.

Marshall LF (2000) Epidemiology and cost of central nervous system injury. Clin Neurosurg 46:105-112.

McIntosh TK, Vink R, Noble L, Yamakami I, Fernyak S, Soares H, Faden AL (1989) Traumatic brain injury in the rat: characterization of a lateral fluid-percussion model. Neuroscience 28:233-244.

McKee JA, Brewer RP, Macy GE, Borel CO, Reynolds JD, Warner DS (2005) Magnesium neuroprotection is limited in humans with acute brain injury. Neurocrit Care 2:342-351.

Mesenge C, Charriaut-Marlangue C, Verrecchia C, Allix M, Boulu RR, Plotkine M (1998) Reduction of tyrosine nitration after N(omega)-nitro-Larginine-methylester treatment of mice with traumatic brain injury. Eur J Pharmacol 353:53-57.

Mohr S, Zech B, Lapetina EG, Brune B (1997) Inhibition of caspase-3 by $\mathrm{S}$-nitrosation and oxidation caused by nitric oxide. Biochem Biophys Res Commun 238:387-391.

Mullen RJ, Buck CR, Smith AM (1992) NeuN, a neuronal specific nuclear protein in vertebrates. Development 116:201-211.

Pike BR, Zhao X, Newcomb JK, Posmantur RM, Wang KK, Hayes RL (1998) 
Regional calpain and caspase-3 proteolysis of alpha-spectrin after traumatic brain injury. NeuroReport 9:2437-2442.

Pike BR, Zhao X, Newcomb JK, Glenn CC, Anderson DK, Hayes RL (2000) Stretch injury causes calpain and caspase- 3 activation and necrotic and apoptotic cell death in septo-hippocampal cell cultures. J Neurotrauma 17:283-298.

Radi R, Beckman JS, Bush KM, Freeman BA (1991) Peroxynitrite oxidation of sulfhydryls. The cytotoxic potential of superoxide and nitric oxide. J Biol Chem 266:4244-4250.

Raghupathi R, Graham DI, McIntosh TK (2000) Apoptosis after traumatic brain injury. J Neurotrauma 17:927-938.

Sattler R, Charlton MP, Hafner M, Tymianski M (1997) Determination of the time-course and extent of neurotoxicity at defined temperatures in cultured neurons using a modified multi-well plate fluorescence scanner. J Cereb Blood Flow Metab 17:455-463.

Sattler R, Charlton MP, Hafner M, Tymianski M (1998) Distinct influx pathways, not calcium load, determine neuronal vulnerability to calcium neurotoxicity. J Neurochem 71:2349-2364.

Sosin DM, Sniezek JE, Thurman DJ (1996) Incidence of mild and moderate brain injury in the United States, 1991. Brain Inj 10:47-54.

Spear N, Estevez AG, Johnson GV, Bredesen DE, Thompson JA, Beckman JS (1998) Enhancement of peroxynitrite-induced apoptosis in PC12 cells by fibroblast growth factor- 1 and nerve growth factor requires p21Ras activation and is suppressed by Bcl-2. Arch Biochem Biophys 356:41-45.

Stefanis L (2005) Caspase-dependent and -independent neuronal death: two distinct pathways to neuronal injury. Neuroscientist 11:50-62.

Sullivan PG, Keller JN, Bussen WL, Scheff SW (2002) Cytochrome c release and caspase activation after traumatic brain injury. Brain Res 949:88-96.

Sun HS, Feng ZP, Miki T, Seino S, French RJ (2006) Enhanced neuronal damage after ischemic insults in mice lacking Kir6.2-containing ATPsensitive K+ channels. J Neurophysiol 95:2590-2601.

Szabo C, Ohshima H (1997) DNA damage induced by peroxynitrite: subsequent biological effects. Nitric Oxide 1:373-385.

Teasdale GM, Graham DI (1998) Craniocerebral trauma: protection and retrieval of the neuronal population after injury. Neurosurgery 43:723-737.

Thurman DJ, Alverson C, Dunn KA, Guerrero J, Sniezek JE (1999) Traumatic brain injury in the United States: a public health perspective. J Head Trauma Rehabil 14:602-615.

Tolias CM, Bullock MR (2004) Critical appraisal of neuroprotection trials in head injury: what have we learned? NeuroRx 1:71-79.

Trackey JL, Uliasz TF, Hewett SJ (2001) SIN-1-induced cytotoxicity in mixed cortical cell culture: peroxynitrite-dependent and -independent induction of excitotoxic cell death. J Neurochem 79:445-455.

Ueta E, Kamatani T, Yamamoto T, Osaki T (2003) Tyrosine-nitration of caspase 3 and cytochrome $\mathrm{c}$ does not suppress apoptosis induction in squamous cell carcinoma cells. Int J Cancer 103:717-722.

Virag L, Marmer DJ, Szabo C (1998) Crucial role of apopain in the peroxynitrite-induced apoptotic DNA fragmentation. Free Radic Biol Med 25:1075-1082.

Volbracht C, Chua BT, Ng CP, Bahr BA, Hong W, Li P (2005) The critical role of calpain versus caspase activation in excitotoxic injury induced by nitric oxide. J Neurochem 93:1280-1292.

Wada K, Chatzipanteli K, Kraydieh S, Busto R, Dietrich WD (1998) Inducible nitric oxide synthase expression after traumatic brain injury and neuroprotection with aminoguanidine treatment in rats. Neurosurgery 43:1427-1436.

Yakovlev AG, Knoblach SM, Fan L, Fox GB, Goodnight R, Faden AI (1997) Activation of CPP32-like caspases contributes to neuronal apoptosis and neurological dysfunction after traumatic brain injury. J Neurosci 17:7415-7424.

Ye YZ, Strong M, Huang ZQ, Beckman JS (1996) Antibodies that recognize nitrotyrosine. Methods Enzymol 269:201-209.

Zipfel GJ, Babcock DJ, Lee JM, Choi DW (2000) Neuronal apoptosis after CNS injury: the roles of glutamate and calcium. J Neurotrauma 17:857-869.

Zou H, Li Y, Liu X, Wang X (1999) An APAF-1.cytochrome c multimeric complex is a functional apoptosome that activates procaspase-9. J Biol Chem 274:11549-11556. 